\title{
MARCACIÓN Y RECAPTURA DEL GATUZO (Mustelus schmitti) EN EL ECOSISTEMA COSTERO BONAERENSE (ARGENTINA)
}

\author{
Marcelo Pérez ${ }^{1,2}$, Matías Braccin ${ }^{3}$ y María B. Cousseau ${ }^{2}$ \\ ${ }^{1}$ Instituto Nacional de Investigación y Desarrollo Pesquero (INIDEP), \\ Paseo Victoria Ocampo No 1, Escollera Norte, B7602HSA - Mar del Plata, Argentina \\ correo electrónico:mperez@inidep.edu.ar \\ ${ }^{2}$ Departamento de Ciencias Marinas, Facultad de Ciencias Exactas y Naturales, \\ Universidad Nacional de Mar del Plata (UNMdP), Funes 3350, B7602AYL - Mar del Plata, Argentina \\ ${ }^{3}$ WA Fisheries and Marine Research Laboratories, 39 Northside Drive Hillarys, WA 6025 Australia
}

RESUMEN. El presente trabajo está referido a los primeros resultados obtenidos sobre marcado y recaptura en el gatuzo, Mustelus schmitti, como medio para reconocer sus desplazamientos y migraciones en el Ecosistema Costero Bonaerense (ECB). Este tiburón es relevante no solo por su gran abundancia a lo largo del ECB sino también por su importancia comercial para la flota pesquera de arrastre argentina. Entre noviembre de 2010 y mayo 2011 se marcaron 2.483 ejemplares cerca de Monte Hermoso, dentro del sector marino conocido como "El Rincón", utilizando como plataforma una embarcación de la flota artesanal. Se logró recuperar 43 individuos entre 2010 y 2013. A partir del análisis de los individuos recapturados se señalaron los patrones de movimientos, migración, distribución espacial y temporal en el ECB, y además se discutieron y relacionaron dichos patrones con el ciclo de vida. Los resultados pusieron en evidencia la utilización por parte del gatuzo de áreas extensas, mayoritariamente costeras, sin alta afinidad con áreas particulares. Este es el modelo tradicional propuesto para tiburones pequeños como el gatuzo. Sin embargo, la modalidad reproductiva indicaría un ajuste al modelo señalado para grandes tiburones. Por lo tanto, el gatuzo en su comportamiento parece contener aspectos correspondientes a ambas modalidades con fidelidad por los sitios de reproducción pero sin evidencias de filopatría.

Palabras clave: Marcas convencionales, Mar Argentino, patrones de movimiento, gatuzo, Mustelus schmitti.

\section{TAG-RECAPTURE OF THE NARROWNOSE SMOOTH-HOUND (Mustelus schmitti) IN BUENOS AIRES COASTAL ECOSYSTEM (ARGENTINA)}

\footnotetext{
ABSTRACT. The present work refers to the first results obtained on tagging and recapture in the Narrownose smooth-hound, Mustelus schmitti, along the Coastal Ecosystem off Buenos Aires Province (BCE) with the aim of describing their migratory movements. This shark is relevant not only because of its high abundance throughout the $\mathrm{BCE}$ but also for its commercial significance for the Argentine trawling fishing fleet. Between November 2010 and May 2011 2,483 specimens were tagged in a marine sector known as "El Rincón", near Monte Hermoso (Buenos Aires Province), using an artisanal fishery vessel belonging to the local fleet as a platform. Fourty-three specimens were recaptured between 2010 and 2013. From the recapture data collected in this study, patterns of movement, migration, spatial and temporal distribution were established and related to the life cycle of the species. Our results evidenced that both juveniles and adults make extensive use of coastal areas, exhibiting low levels of phylopatry. This is the traditional model proposed for small sharks, such as the Narrownose smooth-hound. However, their reproductive aspects would indicate an adjustment to the model indicated for large sharks. Therefore, Narrownose smooth-
} 
hound behaviour would seem to include features corresponding to both modalities, i.e. with fidelity for the breeding sites albeit without evidence of phylopathy.

Key words: Conventional tags, Argentine Sea, movement patterns, Narrownose smooth-hound, Mustelus schmitti.

\section{INTRODUCCIÓN}

La utilización del marcado ha sido reconocida como un valioso procedimiento para estudiar distintos aspectos poblacionales (Hilborn y Walters 1992). Esto incluye desde movimientos y migraciones hasta la estimación de parámetros biológico-pesqueros como el tamaño poblacional, crecimiento somático, sobrevivencia y tasa de explotación (Brownie et al. 1985; Pollock et al. 1991; Hoenig et al. 1998a, 1998b). Existen distintos tipos de marcas, siendo las convencionales las más usadas dados su bajo costo y fácil aplicación. Las marcas convencionales se definen como aquellas que pueden identificarse visualmente en oposición a las que requieren algún otro tipo de instrumental para su utilización (marcas archivos, acústicas y satelitales). La utilización de dichas marcas es una técnica extendida internacionalmente para aportar información relativa a desplazamientos y migraciones (Kohler y Turner 2001).

Los escasos antecedentes disponibles sobre resultados de la aplicación de esta técnica en especies marinas de la Argentina están referidos casi exclusivamente a peces óseos. Como antecedentes en peces cartilaginosos pueden señalarse la marcación de gatuzo Mustelus schmitti Springer, 1939 y el cazón (Galeorhinus galeus), acotada a la zona de Bahía Anegada-San Blas (Cuevas et al. 2013), y de los actuales planes de marcación de grandes tiburones, principalmente para el moteado (Notorynchus cepedianus) y el cazón (Irigoyen et al. 2015). En este trabajo se eligió como especie objetivo al gatuzo por ser el tiburón más abundante y el de mayor interés comercial por parte de la flota pesquera argentina.
M. schmitti es un tiburón demersal-bentónico que alcanza una longitud máxima de $94 \mathrm{~cm}$ (Cousseau 1986; Menni et al. 1986; Cousseau y Perrotta 2013). Es una especie endémica del Atlántico Sudoccidental que se distribuye entre $22^{\circ} \mathrm{S}$ (Brasil) y $47^{\circ} 45^{\prime} \mathrm{S}$ (Argentina), desde zonas costeras hasta los $120 \mathrm{~m}$ de profundidad (Menni et al. 1986) (Figura 1). Al sur de los $34^{\circ} \mathrm{S}$ (límite entre Uruguay y Brasil) las principales concentraciones se encuentran en aguas de las plataformas argentina y uruguaya correspondientes a la Zona Común de Pesca ArgentinoUruguaya (ZCPAU), en aguas de jurisdicción provincial y en la plataforma bonaerense hasta los $42^{\circ} \mathrm{S}$ aproximadamente, por fuera de la ZCPAU (Figura 1). Esta especie es capturada principalmente por parte de la flota argentina (alrededor de $4.000 \mathrm{t}$ anuales durante los últimos años) en la pesquería multiespecífica del área costera de la Provincia de Buenos Aires.

Aunque no se ha realizado aún una evaluación de stock formal de la especie para la totalidad de su área de distribución en la Argentina, a partir de 2011 y dentro del ámbito del área del Tratado del Río de la Plata y su Frente Marítimo, se han elaborado evaluaciones mediante modelos de producción excedente. Como resultado, las biomasas estimadas indicaron un decrecimiento sostenido (Cortés et al. 2016) y la biomasa total a comienzos de 2016 fue entre un $43 \%$ y un $64 \%$ menor con respecto al nivel correspondiente al año inicial del período de evaluación (1983). El gatuzo ha sido incorporado a la lista roja de la Unión Internacional para la Conservación de la Naturaleza (UICN 2015) como especie "en peligro", con indicadores de disminución de la abundancia en su área de distribución, con un importante impacto por efecto de la pesca en aguas de Brasil y con la mención de la necesidad de enten- 
der sus patrones migratorios para sugerir medidas pertinentes de ordenación pesquera (Massa et al. 2006).

En trabajos previos, tanto a partir de datos biológicos colectados en campañas de investigación como los originados a partir de muestreos de los desembarques de las flotas comerciales, se han discutido e hipotetizado los potenciales desplazamientos del gatuzo vinculados con cambios estacionales de la estructura poblacional y de los parámetros reproductivos en la Argentina (Menni et al. 1986; Cousseau et al. 1998; Massa et al. 2004; Cortés et al. 2011), Uruguay (Oddone et al. 2007; Pereyra et al. 2008) y Brasil (Vooren 1997). En el marco del Programa "Pesquerías de Con- drictios" del Instituto Nacional de Investigación y Desarrollo Pesquero (INIDEP), se diseñó un plan para la utilización de la técnica de marcado y recaptura en el gatuzo con la finalidad de poder reconocer patrones de movimiento de la especie en el Ecosistema Costero Argentino-Uruguayo (Pérez y Massa 2011). El presente trabajo es una descripción de los resultados de dicho plan que también son los primeros disponibles para esta especie mediante la aplicación de la técnica de marcas convencionales. A partir de 2016, y con objetivos similares, se estableció en la ZCPAU un plan de marcación de gatuzo conjunto entre Argentina y Uruguay actualmente en desarrollo (Silveira et al. 2018).

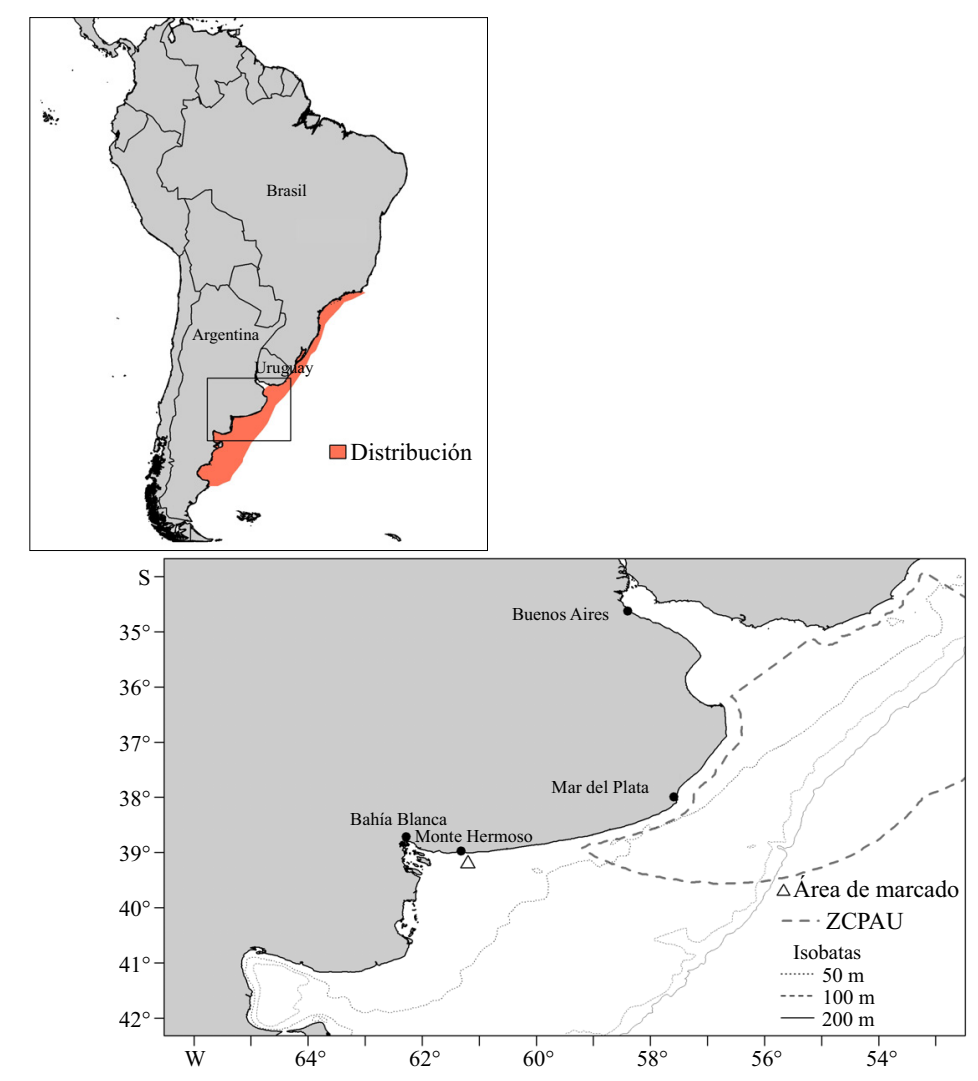

Figura 1. Distribución del gatuzo en el Atlántico Sudoccidental con detalle del área de trabajo en el litoral de la Provincia de Buenos Aires, Argentina. Zona de marcado de ejemplares de gatuzo (triángulo) frente a Monte Hermoso, Provincia de Buenos Aires, Argentina.

Figure 1. Narrownose smooth-hound distribution in the Southwestern Atlantic indicating the working area. Tagging area (triangle) in front of Monte Hermoso, Buenos Aires Province, Argentina. 


\section{MATERIALES Y MÉTODOS}

\section{Área de estudio}

Características oceanográficas a lo largo de la costa de la Provincia de Buenos Aires

En esta zona la temperatura es función de la profundidad y de la latitud, con variaciones importantes en el plano vertical, mientras que la salinidad puede ser considerada como indicador más conservativo para caracterizar las distintas masas de agua presentes estacionalmente. Debido a esto, las masas de agua se clasificaron según su salinidad mediante el criterio de Lucas et al. (2005):

- Mínima salinidad debido a la descarga del Río de la Plata $(0-33,0)$.

- Aguas características de baja salinidad de "El Rincón" (30,0-33,3).

- Máxima salinidad, aguas dentro y boca del Golfo San Matías $(33,9-34,1)$ y área muy costera cercana al este de "El Rincón" (33,7-34,1). - Aguas de plataforma continental $(33,5-33,7)$.

\section{Sistemas frontales}

Acha et al. (2004) describieron los frentes presentes en el Mar Argentino y señalaron las características particulares de las áreas frontales del Río de la Plata y de "El Rincón". El primero está caracterizado por una fuerte estratificación vertical con agua de baja salinidad originada por la descarga del río en las capas más superficiales y el agua salada en las capas más profundas en forma de cuña. Se ubica al sur de Punta Rasa (Provincia de Buenos Aires) entre los $35^{\circ} \mathrm{S}$ y $37^{\circ} \mathrm{S}$. El segundo corresponde al sur de la Provincia de Buenos Aires, en la costa de "El Rincón" (39 $\mathrm{S}$ a $41^{\circ} \mathrm{S}$, profundidades menores a $40 \mathrm{~m}$ ) con orientación norte-sur, y se caracteriza por tener aguas homogéneas verticalmente debido al forzante de las mareas, entre aguas diluidas correspondientes a los aportes de los ríos próximos (Negro y Colorado) por "dentro" (inshore) y aguas de alta salinidad originadas en el Golfo San Matías por "fuera" (offshore).

\section{Ecosistema Costero Bonaerense}

Está definido por el sector bonaerense desde la línea de costa hasta la isobata de $50 \mathrm{~m}$ de profundidad al este, y desde $34^{\circ} \mathrm{S}$ en el norte hasta los $42^{\circ} \mathrm{S}$ en el sur. Forma parte del mismo el área denominada "El Rincón" cuya delimitación aproximada es entre $38^{\circ} 40^{\prime} \mathrm{S}$ y $41^{\circ} \mathrm{S}$. En este ecosistema la fauna presenta características propias que en el caso de los peces está dominada por los sciénidos y otras especies (algunas de peces cartilaginosos como el gatuzo) que desde un punto de vista comercial u operativo se conoce como "variado costero", sobre el cual desarrolla sus actividades la flota pesquera costera fresquera marplatense (Lasta et al. 2001).

\section{Marcación de ejemplares y variables conside- radas}

Entre noviembre de 2010 y diciembre de 2011 se marcaron 2.483 ejemplares de gatuzo mayores de $50 \mathrm{~cm}$ de longitud total, mediante marcas convencionales tipo DART (Hallprint, PDS small plastic tipped tags) con "cabeza" de plástico con un largo del tubo de $10 \mathrm{~cm}$. La metodología y proceso de marcación en Monte Hermoso (Provincia de Buenos Aires, Argentina), en conjunto con el plan de divulgación para la recuperación de marcas, fueron presentados en Pérez et al. (2014). En ese trabajo también se describen las cuestiones operativas inherentes al proceso de marcado, la descripción del origen de los reportes de ejemplares marcados, el criterio de asignación de posiciones (criterio de mínima distancia) y fechas de recapturas mediante la utilización de los Partes de Pesca en los que se detectaron marcas en las plantas de procesamiento de pescado.

La marcación se realizó en cuatro campañas o comisiones realizadas en noviembre de 2010 
(1.199 individuos marcados) y en marzo, mayo y diciembre de 2011 (599, 550 y 135 individuos marcados respectivamente). De la totalidad de los ejemplares marcados se dispuso del número de identificación de la marca (Nro_marca), la fecha en el que fue marcado como el día, mes y año (Comisión: mes y año), la longitud total (talla_cap_cm, variable continua), la posición de marcado (Latitud y Longitud) y el sexo (Tabla 1).

Hasta diciembre de 2012 se habían recuperado 42 marcas. Luego en 2013 fue declarada una marca adicional, por lo que el número total de ejemplares recapturados fue de 43 . No hubo nuevos reportes con posterioridad. De este total, 3 ejemplares no fueron considerados para este análisis debido a la carencia de las posiciones en las declaraciones de recapturas respectivas. Por lo tanto, la base de datos a analizar fue construida con 40 ejemplares (Tabla 1). De cada ejemplar recapturado se obtuvo: la posición asignada a cada recaptura (Latitud y Longitud), el origen de la declaración de la recaptura (Origen recaptura y origen_declar), la fecha asignada y el mes de recaptura (mes_recaptura) (Tabla 1). A partir de estos datos se estimaron las siguientes variables: el tiempo de recaptura expresado en días (Tiempo_recaptura_días, variable continua), el rumbo verdadero (rumbo, variable continua) expresado en grados (de 0, rumbo Norte, a 360) y la distancia (distancia, variable continua expresada en millas náuticas) entre las posiciones de marcado y de recaptura estimados mediante las funciones bearingRhumb y distrumb del entorno estadístico R (v 3.0.2) (R Core Team 2013) (Tabla 1). Además, la profundidad asignada a cada posición de recaptura (Profundidad, variable continua) en metros obtenida de una base de datos internacional (Becker et al. 2009; Scripps 2014). Por otra parte, la velocidad de natación fue estimada como la distancia entre la posición de marcación y la de recaptura, dividida por el tiempo en libertad.

Mediante el test de Kolmogorov-Smirnov (KS) se compararon las tallas al momento del marcado, entre los individuos que fueron y no fueron recap- turados. El test de Chi cuadrado se utilizó para evaluar las diferencias en la proporción de sexos del total de los individuos recapturados. Con el test espacial de Rao (1967) se evaluó la uniformidad en la distribución de las direcciones de desplazamiento o si existió alguna direccionalidad sesgada, al utilizar Estadística Circular Estándar (Zar 1999), mediante el mismo entorno estadístico R.

Se consideró la temperatura superficial correspondiente al día y posición de marcación y de recaptura (ssts_marcado y ssts_recap, variables continuas) con las que también se estimó la diferencia entre ambas (gradiente de temperatura, gradtemp, variable continua), disponibles en la base de datos de la agencia gubernamental NOAA de EE. UU. (NOAA 2014) (Tabla 1). Para la caracterización de las masas de agua presentes en la zona se estimó la salinidad de superficie (sal recap, variable continua) como un promedio histórico mensual en una grilla de $0,5^{\circ}$ de Latitud y Longitud que incluyó las posiciones y el mes de cada recaptura correspondientes a observaciones realizadas en campañas de investigación del INIDEP (INIDEP 2015). Dada la proximidad a la costa y a la falta de observaciones en algunos meses del año, no pudo asignarse un valor de salinidad a tres marcas recuperadas, por lo tanto se la consideró solo en la discusión.

Las relaciones entre las variables estimadas a partir del marcado y recaptura (Tabla 1) se presentan en la Figura 2. Para detectar posibles relaciones (agrupamiento) entre los individuos recuperados se realizaron análisis multivariados: Agrupamiento Jerárquico Aglomerativo (Análisis de cluster, agglomerative hierarchical clustering) y Análisis de Componentes Principales (ACP). En ambos fueron consideradas como variables continuas el gradiente de temperatura (gradtemp), el tiempo de recaptura, el rumbo, la profundidad y la distancia. El Agrupamiento Jerárquico Aglomerativo fue realizado mediante la distancia euclídea con las variables estandarizadas y el promedio como enlace mediante la función agnes del 


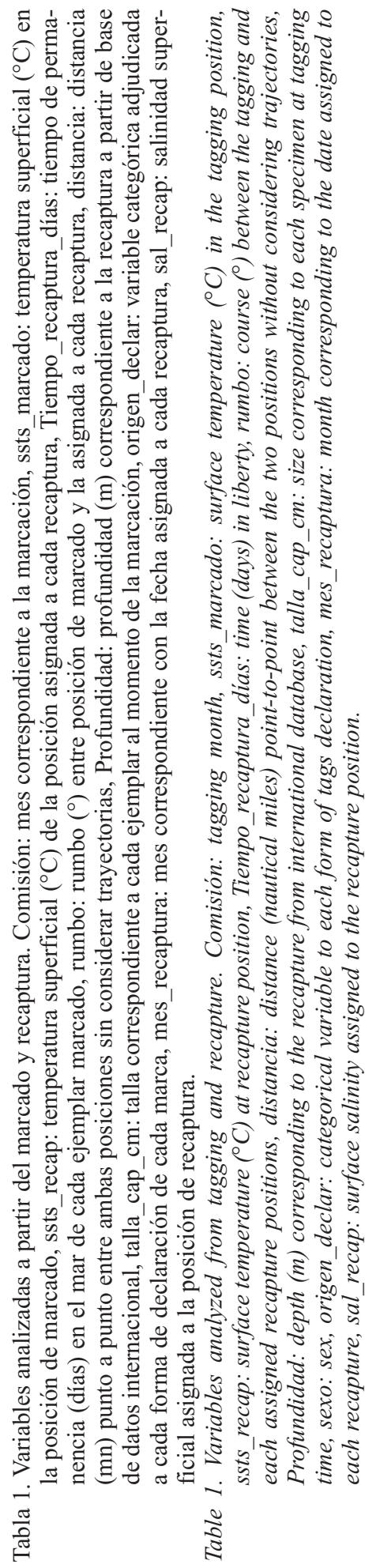

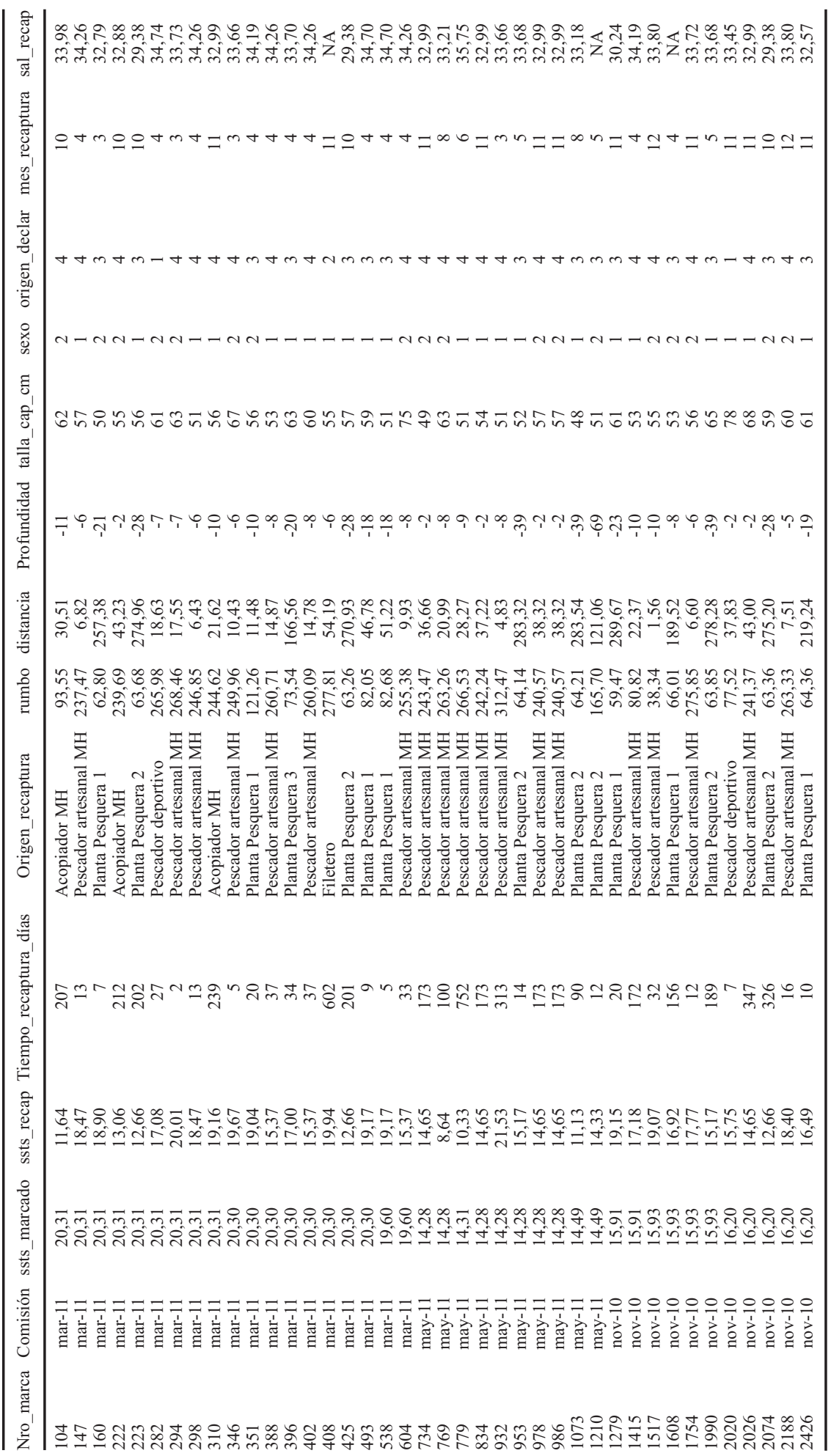




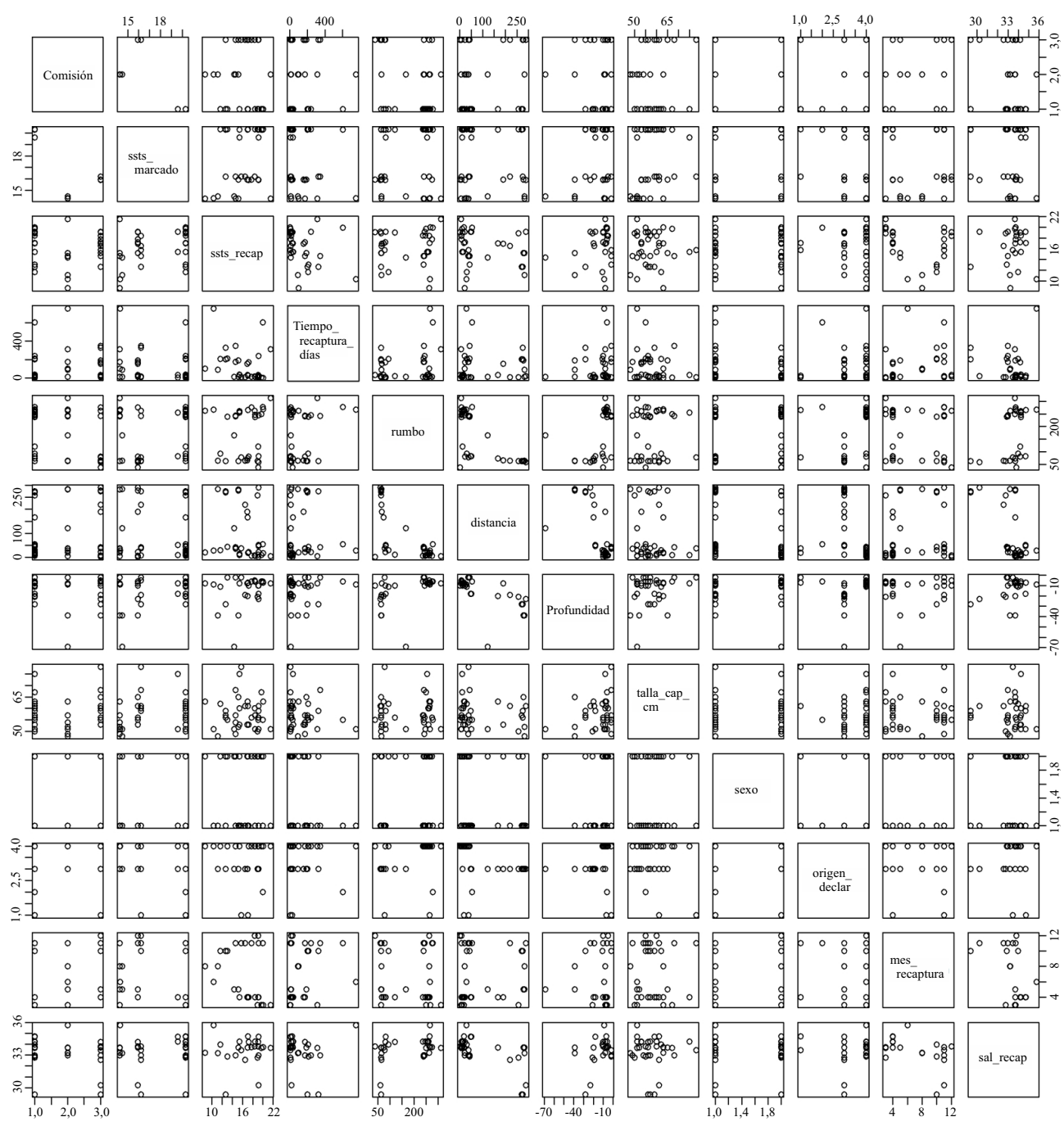

Figura 2. Relaciones entre variables estimadas a partir del marcado y recaptura. Comisión: mes correspondiente a la marcación, ssts_marcado: temperatura superficial $\left({ }^{\circ} \mathrm{C}\right)$ en la posición de marcado, ssts_recap: temperatura superficial $\left({ }^{\circ} \mathrm{C}\right)$ de la posición asignada a cada recaptura, Tiempo_recaptura_días: tiempo de permanencia (días) en el mar de cada ejemplar marcado, rumbo: rumbo $\left(^{\circ}\right)$ entre posición de marcado y la asignada a cada recaptura, distancia: distancia (mn) punto a punto entre ambas posiciones sin considerar trayectorias, Profundidad: profundidad $(\mathrm{m})$ correspondiente a la recaptura a partir de base de datos internacional, talla_cap_cm: talla $(\mathrm{cm})$ correspondiente a cada ejemplar al momento de la marcación, sexo, origen_declar: variable categórica adjudicada a cada forma de declaración de cada marca, mes_recaptura: mes correspondiente con la fecha asignada a cada recaptura, sal_recap: salinidad superficial asignada a la posición de recaptura.

Figure 2. Relationships between variables estimated from the mark-recapture. Comisión: tagging month, ssts_marcado: surface temperature $\left({ }^{\circ} \mathrm{C}\right)$ in the tagging position, ssts_recap: surface temperature $\left({ }^{\circ} \mathrm{C}\right)$ recapture position, Tiempo_recaptura_dias: time (days) in liberty, rumbo: course $\left(^{\circ}\right)$ between the tagging position and the one each recapture assigned, distancia: distance (nautical miles) point-to-point between the two positions without considering trajectories, Profundidad: depth $(\mathrm{m})$ corresponding to the recapture from international database, talla_cap_cm: size (cm) corresponding to each specimen at tagging time, sex, origen_declar: categorical variable to each form of tags declaration, mes_recaptura: month corresponding with the date assigned to each recapture, sal_recap: surface salinity assigned to the recapture position. 
paquete de programas estadísticos $\mathrm{R}$ ( $\mathrm{R}$ Core Team 2013). El ACP fue realizado mediante la función prcomp del mismo programa con las variables logaritmizadas (logaritmo natural, aunque en todos los casos expresadas como $\log$ ) y estandarizadas. Aunque correlacionadas, se conservaron la profundidad, el rumbo y la distancia, relevantes para la descripción de los desplazamientos. La talla correspondió a la estimada al momento del marcado, ya que en la mayoría de los casos no se la declaró con la recaptura. Por lo tanto, no se la consideró para estos análisis, pero sí se la incluyó luego en la discusión. De todas maneras, la talla no se correlacionó con ninguna de las variables restantes (Figura 3).

Se construyó un Modelo Líneal Generalizado (MLG) con distribución lognormal del término de error, con la distancia como variable respuesta y como explicativas, el resto de las consideradas en los análisis anteriores. Fueron logaritmizadas las variables continuas Tiempo_recaptura_días y talla_cap_cm. Profundidad y rumbo no fueron incorporadas debido a las correlaciones detectadas entre estas y la variable respuesta. Además de los factores sexo y mes_recaptura, se incorporó también el mes_marcado equivalente a la variable Comisión (Tabla 1). Este modelo también fue construido mediante el paquete de programas estadísticos R (R Core Team 2013). El MLG diseñado fue:

$$
\begin{aligned}
& \log \left(\text { distancia }_{i}\right)=\mu+\text { sexo }_{i}+\text { mes_marcado }{ }_{i} \\
& + \text { mes_recaptura }_{i}+\text { gradtemp }_{i} \\
& +\log \left(\text { Tiempo_recaptura_dias }{ }_{i}\right) \\
& +\log (\text { talla_cap_cm })_{i}+\varepsilon_{i}
\end{aligned}
$$

donde:

$\log \left(\right.$ distancia $\left._{i}\right)=$ logaritmo natural de la distancia $(\mathrm{mn})$ entre la posición de marcado y la asignada de recaptura para cada individuo $i$;

$\mu=$ constante del modelo;

$\varepsilon_{i}=$ término de error del modelo.

\section{Tiempos de permanencia}

En aquellos casos cuya recaptura se produjo en rumbos menores a $122^{\circ}$, y para evitar sesgos en las trayectorias punto a punto que podrían atravesar partes terrestres, se las forzó a pasar por dos posiciones arbitrarias. La primera fue $39^{\circ} \mathrm{S}$ $59^{\circ} \mathrm{W}$ considerada como la primera posición dentro de la Zona Común de Pesca ArgentinoUruguaya (ZCPAU). La restante fue un punto al sur de la ciudad de Mar del Plata (38 $38^{\circ} \mathrm{S}-57^{\circ}$ $\left.24^{\prime} \mathrm{W}\right)$ para permitir "girar" las trayectorias debido a la forma de la costa de la Provincia de Buenos Aires. En función de este criterio se estimaron nuevamente las distancias correspondientes a los 18 ejemplares recapturados en este rango de rumbos, dentro y fuera de la ZCPAU. Luego las distancias se consideraron proporcionales al tiempo de recaptura.

\section{RESULTADOS}

\section{Desplazamientos, distribución espacial y tem- poral a partir de la recaptura de ejemplares marcados}

Debido a las dificultades propias del método de marcado con marcas convencionales, es decir, la posibilidad de pérdida inmediata de marcas, la falta de reportes de marcas recuperadas y los efectos de las mortalidades naturales y por pesca, los modelos fueron ajustados con los individuos recapturados (McGarvey y Feenstra 2002). El test de KS manifestó una diferencia $\left(\mathrm{p}=2,4410^{-7}\right)$ entre la distribución de tallas al momento de la marcación de los individuos recapturados (40 individuos) respecto de aquellos no recapturados (2.483 individuos). Esto fue debido posiblemente al bajo número de recapturas. Por lo tanto, como aporte, los análisis se realizaron bajo el supuesto de representatividad de los individuos recapturados. 


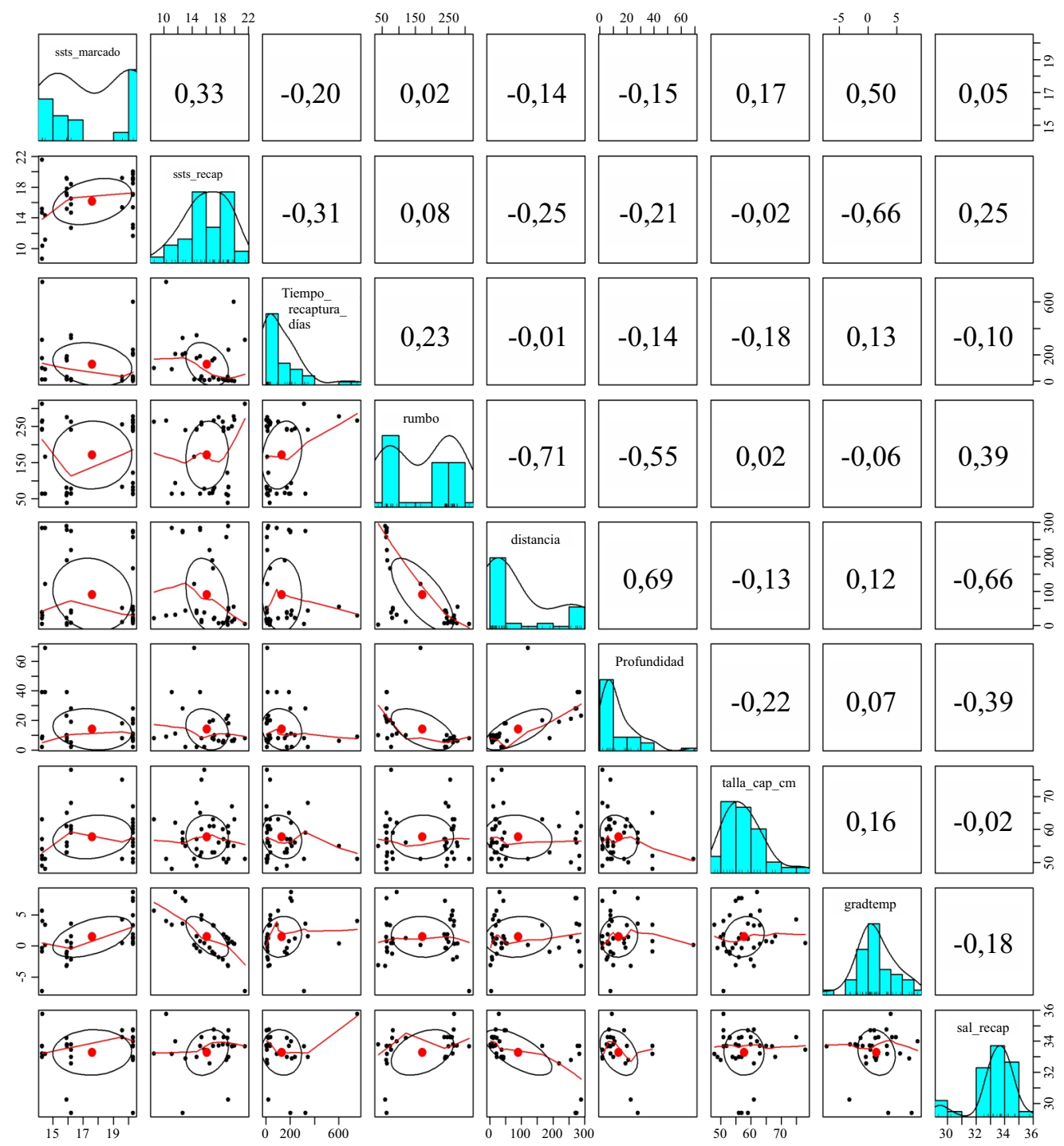

Figura 3. Distribución y correlaciones entre las variables continuas disponibles en la base de datos. Ssts_marcado: temperatura $\left({ }^{\circ} \mathrm{C}\right)$ superficial posición de marcado, ssts_recap: temperatura $\left({ }^{\circ} \mathrm{C}\right)$ superficial de la posición asignada a cada recaptura, Tiempo_recaptura_días: tiempo de permanencia en el mar de cada ejemplar marcado, rumbo: rumbo $\left(^{\circ}\right)$ verdadero entre posición de marcado y la asignada para cada recaptura, distancia: distancia ( $\mathrm{mn}$ ) punto a punto entre ambas posiciones sin considerar trayectorias, Profundidad: profundidad $(\mathrm{m})$ correspondiente a la recaptura obtenida a partir de base de datos internacional, talla_cap_cm: talla correspondiente a cada ejemplar al momento de la marcación, gradtemp: diferencias entre la temperatura $\left({ }^{\circ} \mathrm{C}\right)$ asignada a la posición de marcado y la correspondiente a la recaptura, sal_recap: salinidad superficial asignada a la posición de recaptura.

Figure 3. Distribution and correlations between continuous variables available in the database. Ssts_marcado: surface temperature $\left({ }^{\circ} \mathrm{C}\right)$ in the tagging position, ssts_recap: surface temperature $\left({ }^{\circ} \mathrm{C}\right)$ recapture position, Tiempo_recaptura_dias: time (days) in liberty, rumbo: course $\left(^{\circ}\right)$ between the tagging position and the one each recapture assigned, distancia: distance (nautical miles) point-to-point between the two positions without considering trajectories, Profundidad: depth (m) corresponding to the recapture from international database, talla_cap_cm: size corresponding to each specimen at tagging time, gradtemp: differences between the temperature $\left({ }^{\circ} \mathrm{C}\right)$ assigned to the position of tagging and the corresponding to the recapture, sal_recap: surface salinity assigned to the recapture position. 
Hubo recapturas en 8 meses del año (marzo, abril, mayo, junio, agosto, octubre, noviembre y diciembre) aunque la mayor proporción correspondió a los meses de primavera (18, entre octubre y diciembre) y otoño (19, entre marzo y mayo) (ver Figura 2). En invierno (agosto) se recapturaron 2 individuos. No hay una clara relación entre el momento de marcación, el mes de recaptura y el rumbo respecto del tiempo en libertad transcurrido. Tampoco entre estas últimas variables con la talla y el sexo de los ejemplares.

Las posiciones de recaptura de los 40 individuos marcados en Monte Hermoso en noviembre de 2010, marzo y mayo de 2011, objeto de este análisis, abarcaron el área costera, al este de Cabo San Antonio, hasta la boca del Golfo San Matías, aproximadamente entre $36^{\circ} 30^{\prime} \mathrm{S}$ y $41^{\circ} 10^{\prime} \mathrm{S}$ (Figura 4). Las profundidades correspondientes a las posiciones de las recapturas fueron estimadas entre 2 (cercanas a la línea de costa) y $69 \mathrm{~m}$, esta última correspondiente al individuo recapturado frente a la boca del Golfo San Matías. Puede observarse también que, con excepción del último individuo mencionado, el resto (39 individuos) fue recapturado en profundidades menores a $50 \mathrm{~m}$.

De los 40 individuos recapturados, 11 (27,5\%) lo fueron al norte o en zonas cercanas a la ciudad de Mar del Plata (Zona Norte) (Figura 4). El resto lo fue en áreas cercanas a Monte Hermoso (Zona Sur), un caso dentro de la Ría de Bahía Blanca y un caso frente a la boca del Golfo San Matías (Figura 4). El número y el porcentaje del total de las recapturas también se estimó considerando como unidad espacial el rectángulo estadístico de un grado de Latitud por uno de Longitud (RE) (Tabla 2). El RE con mayor número de recapturas (21; 52,5\% del total) fue el 3961 dentro de la Zona Sur ("El Rincón"). El siguiente en cuanto a las recapturas fue el 3960 (3; 7,5\%). En el norte, fueron el $3655(4 ; 10 \%)$ y el $3755(3 ; 7,5 \%)$ (Figura 5).

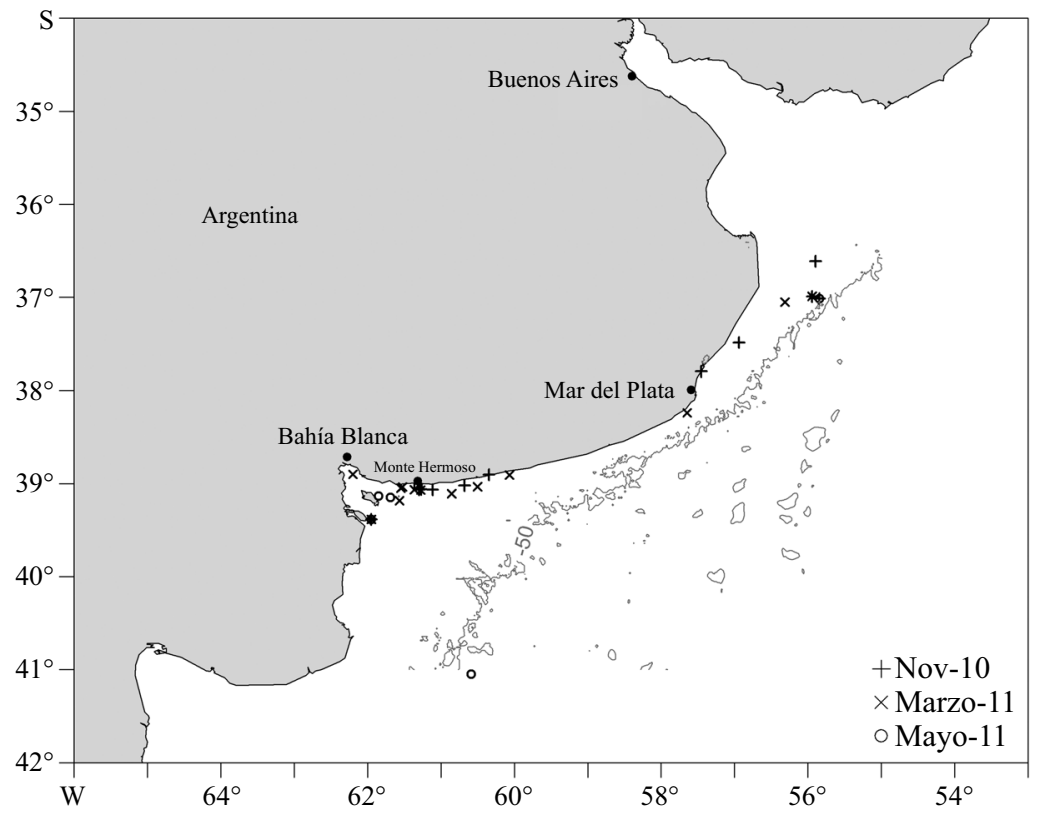

Figura 4. Posiciones asignadas a cada recaptura según el mes de marcación, noviembre de 2010 (Nov-10), marzo de 2011 (Marzo-11) y mayo de 2011 (Mayo-11). La línea continua gris representa la isobata de $50 \mathrm{~m}$ de profundidad.

Figure 4. Positions assigned to each recapture according to the tagging month, November 2010 (Nov-10), March 2011 (Marzo11) and May 2011 (Mayo-11). The solid gray line represents the $50 \mathrm{~m}$ isobath. 
Tabla 2. Número y porcentaje de individuos recapturados totales por rectángulo estadístico de un grado de Latitud por uno de Longitud (RE).

Table 2. Number and percentage of total recaptured individuals by statistical rectangle of one degree of Latitude by one of Longitude (RE).

\begin{tabular}{lcc}
\hline RE recaptura & Número de recapturas & \% recapturas \\
\hline 3655 & 4 & 10,0 \\
3755 & 3 & 7,5 \\
3756 & 2 & 5,0 \\
3757 & 1 & 2,5 \\
3857 & 1 & 2,5 \\
3860 & 3 & 7,5 \\
3862 & 1 & 2,5 \\
3960 & 3 & 7,5 \\
3961 & 21 & 52,5 \\
4160 & 1 & 2,5 \\
\hline Total & 1 & 100,0 \\
\hline
\end{tabular}

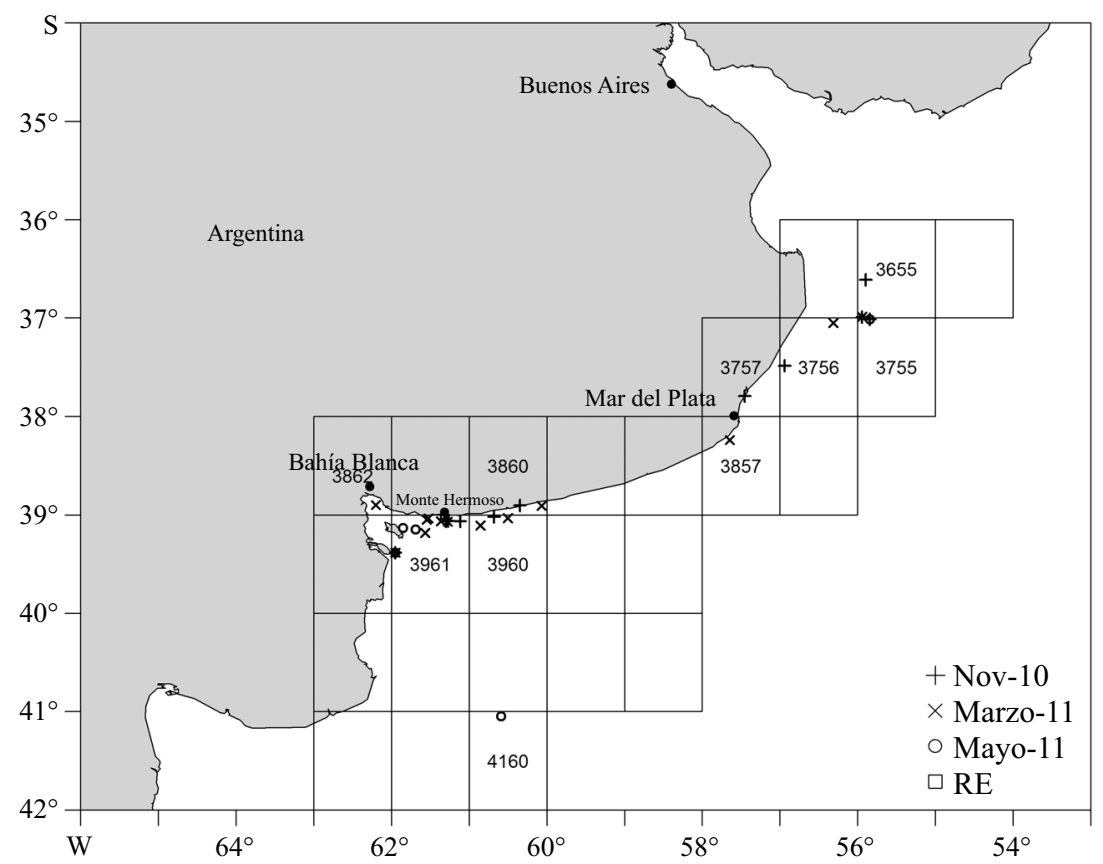

Figura 5. Posiciones de recapturas por rectángulo estadístico (RE) según el mes de marcación. Los RE sin recapturas figuran en blanco.

Figure 5. Positions of recaptures by statistical rectangle (RE) according to the tagging month. The RE without recaptures are contained in white. 
Existen principalmente dos tendencias mayoritarias en los rumbos; menores a $100^{\circ}$ (con mayoría de valores entre $50^{\circ}$ y $100^{\circ}$ ), y aquellos entre $225^{\circ}$ y $300^{\circ}$ (Figura 6). En función de este criterio se calcularon los promedios de $68,5^{\circ}$ (rumbo ENE) y $254^{\circ}$ (rumbo OSO). De los 40 individuos recapturados, $17(43 \%)$ correspondieron a rumbos promedio ENE. 20 individuos (50\%) fueron recapturados con rumbo OSO y los tres ejemplares restantes lo fueron en otros rumbos.

El rango de distancias de recaptura, estimadas punto a punto entre la posición de marcado y la de recaptura, fue entre 1,56 y $289,67 \mathrm{mn}$, equivalentes a 2,89 y 536,46 km, respectivamente. Las mayores distancias correspondieron a rumbos ENE, aunque estuvieron subestimadas debido a la imposibilidad de determinar la trayectoria de cada ejemplar entre el lugar de marcación y las posiciones de recapturas correspondientes a la Zona Norte, ya que necesariamente los individuos debieron ir hacia el este y "rodear" la costa de la provincia de Buenos Aires. Con una trayectoria supuesta, considerando una poligonal entre la posición de marcado, el extremo este de la provincia como punto geográfico y las posiciones de recaptura, la mayor distancia estimada fue $580 \mathrm{~km}$.

Los tiempos de recaptura variaron entre 2 y 752 días. Hasta los 400 días se recapturaron ejemplares en ambos rumbos promedio. Los dos ejemplares con mayores tiempos de recaptura (602 y 752 días) fueron machos, cuyas distancias de recapturas fueron menores a $55 \mathrm{mn}$, con posiciones que correspondieron a rumbos OSO (Figura 7).

Para conocer el potencial efecto del momento de la marcación (noviembre 2010 y marzo y mayo 2011) respecto de las posiciones y tiempos de recaptura, se graficaron los tiempos de recaptura expresados en días correspondientes a los individuos marcados en noviembre de 2010 (Figura 8 A). Se recapturaron individuos en un

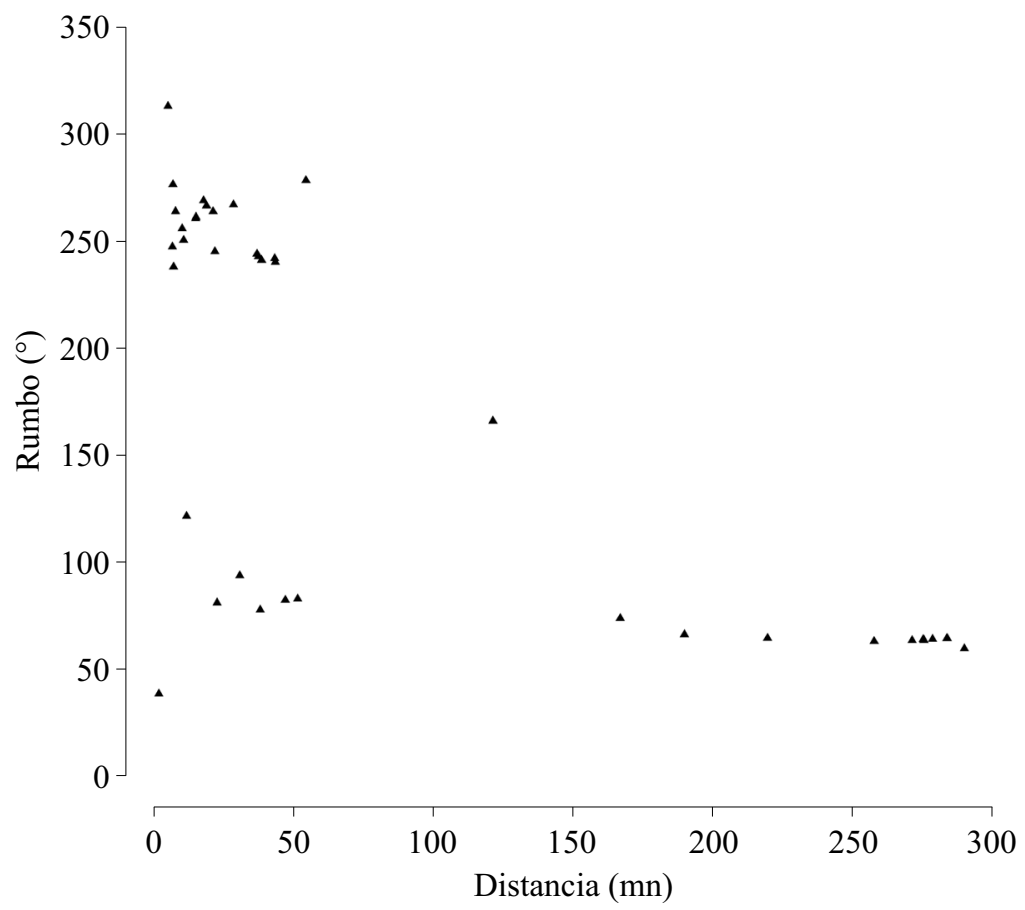

Figura 6. Relación entre el rumbo y la distancia. Ambas estimaciones consideradas entre la posición de marcado y recaptura. Figure 6. Relationship between the course and the distance. Both estimates considered between the tagging and recapture position. 


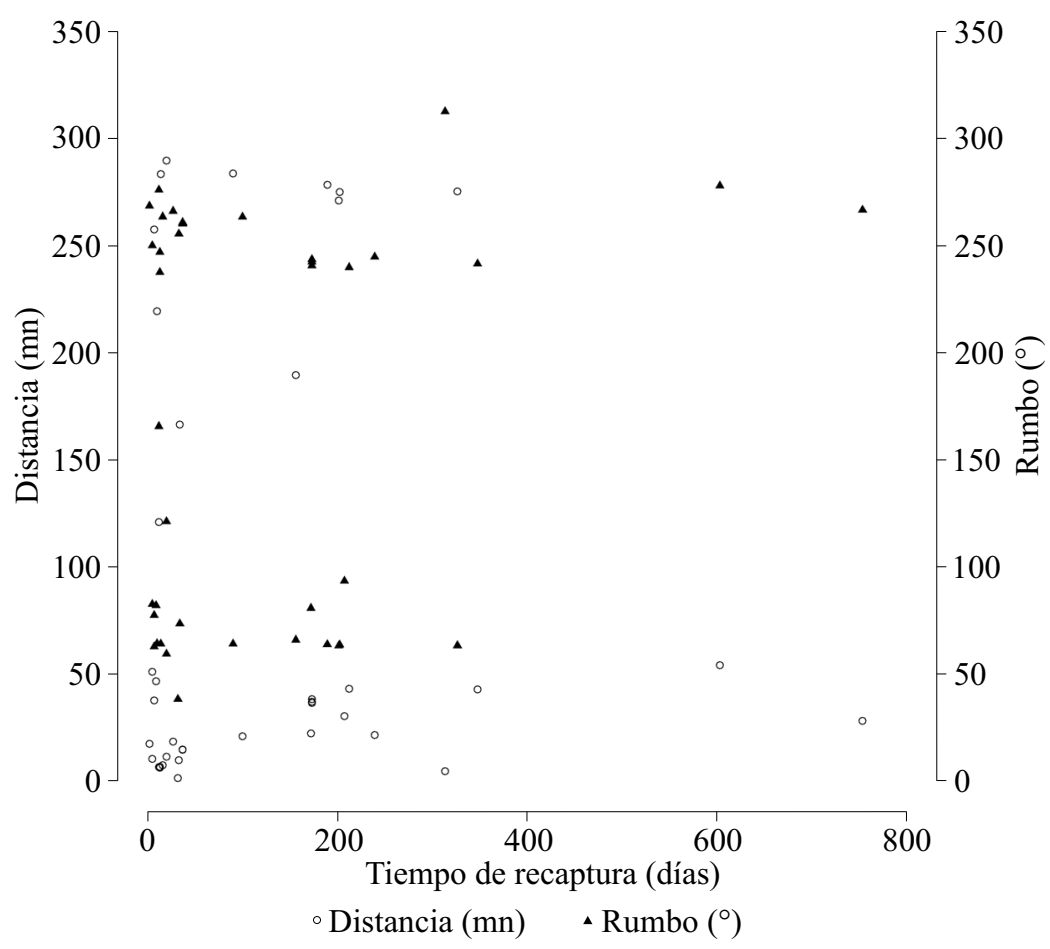

Figura 7. Distancia y rumbo (eje secundario), entre la posición de marcado y recaptura, versus tiempo de recaptura. Figure 7. Distance ( $\mathrm{mn}$ ) and course (secondary axis), between the tagging and recapture position, versus recapture time.

rango de 7 a 347 días de permanencia en libertad. Existieron casos con pocos días (entre 7 y 32) y con muchos (más de 100) recapturados tanto al norte de Mar del Plata como en la zona cercana a Monte Hermoso. Para los marcados en marzo de 2011, se observa un patrón similar (Figura 8 B). Los tiempos de recaptura fueron entre 2 y 602 días similares en ambas zonas. Los marcados en mayo 2011 presentan la misma tendencia que en los casos anteriores, se observan dos ejemplares con características particulares, uno con el mayor tiempo de recaptura (752 días) y el único ejemplar recapturado frente al norte del Golfo San Matías con 12 días de permanencia en libertad (Figura $8 \mathrm{C}$ ).

Respecto de la velocidad de natación, para disminuir la variabilidad debido a la imposibilidad de medir la trayectoria en aquellos casos de recapturas al norte o frente a la costa de Mar del Plata, la estimación de velocidades se realizó sin ellos (subconjunto remanente de 29 individuos). En este subconjunto, el rango de velocidades estuvo comprendido entre 0,03 y $19 \mathrm{~km} \mathrm{día}^{-1}$, con un promedio de $3,1 \mathrm{~km} \mathrm{día}^{-1}$. Sin embargo, en dos casos con los menores tiempos de recaptura (2 y 7 días) que fueron considerados de alta confiabilidad en la declaración de la fecha y posición (pescador artesanal de Monte Hermoso y de un pescador deportivo), la estimación fue de 16 y 10 $\mathrm{km}$ día $^{-1}$ respectivamente. Las mayores velocidades estimadas (19 $\left.\mathrm{km} \mathrm{día}^{-1}\right)$, correspondieron a un macho y una hembra con la misma talla $(51 \mathrm{~cm})$. Las menores $\left(0,03\right.$ y $\left.0,07 \mathrm{~km} \mathrm{día}^{-1}\right)$ también correspondieron a dos machos de la misma talla. De los 29 individuos solo 6 desarrollaron velocidades superiores a los $3,5 \mathrm{~km} \mathrm{día}^{-1}$. Con el resto fueron menores a $1,3 \mathrm{~km}$ día ${ }^{-1}$.

Los individuos marcados en noviembre fueron recapturados en casi las mismas condiciones de temperatura superficial en la que fueron marca- 

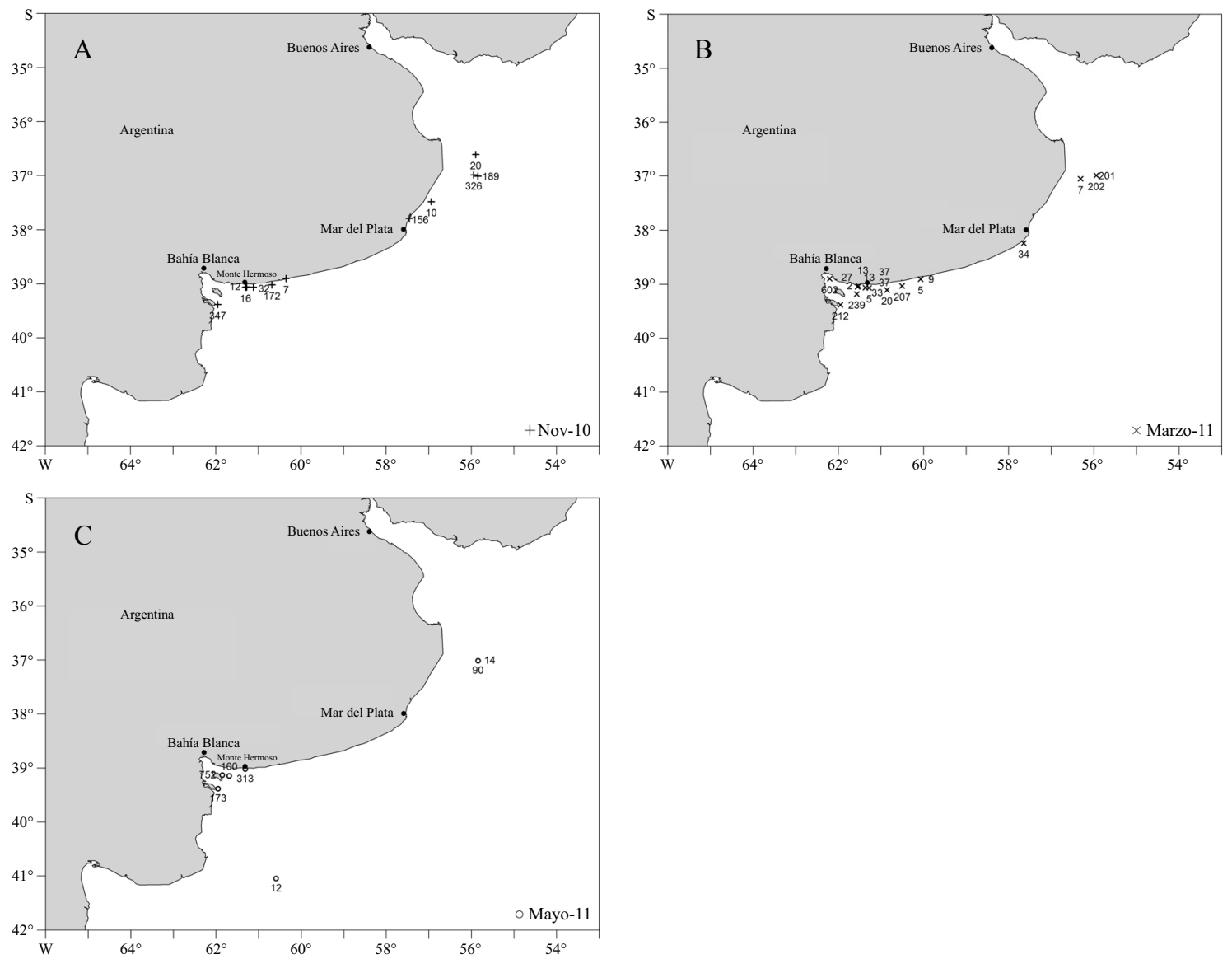

Figura 8. Posiciones de recaptura y días de permanencia en el mar de los individuos marcados en noviembre de 2010 (A), en marzo (B) y en mayo de 2011 (C).

Figure 8. Recapture positions and days in liberty at sea of individuals tagged in November 2010 (A), March (B) and May 2011 (C).

dos. Los marcados en mayo y marzo fueron recapturados en zonas con menores temperaturas superficiales respecto de las imperantes al momento de la marcación (Figura 9), con la excepción de un individuo marcado en mayo que fue recapturado frente a Monte Hermoso muy cerca de la costa, con temperaturas muy superiores a la correspondiente al momento de la marcación (Nro_marca 932, Tabla 1).

Las recapturas en aguas de baja salinidad de superficie, tanto en "El Rincón" como en el área de descarga del Río de la Plata, indican que los individuos fueron recapturados en áreas "fronta- les" (Figura 10). El resto mayoritariamente fue recapturado en altas salinidades correspondientes a aguas costeras de "El Rincón" y con influencia de aguas del Golfo San Matías. En la Tabla 1 puede observarse que al ejemplar recapturado en la boca del Golfo San Matías, no pudo asignársele una salinidad de superficie. Sin embargo, en función de lo descripto por Lucas et al. (2005) y Matano et al. (2014), en dicha zona también se encontrarían dos masas de agua, una de alta salinidad superficial correspondiente al Golfo San Matías por el oeste y por el este Aguas de Plataforma Continental. 


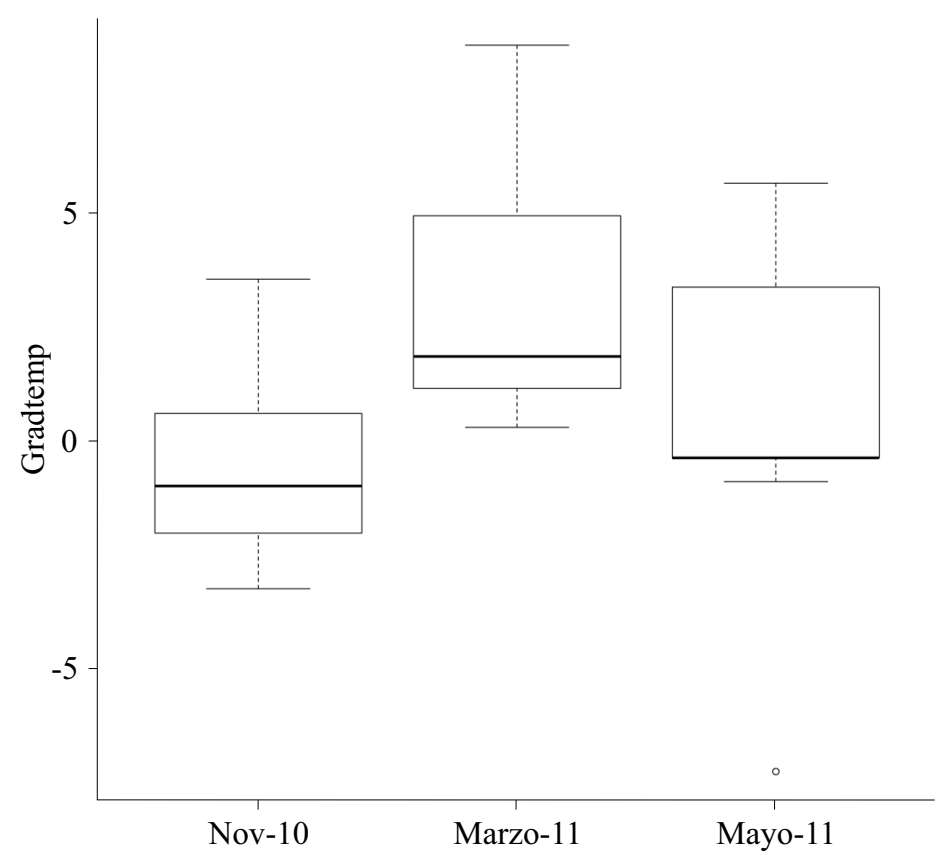

Figura 9. Boxplot con las diferencias entre la temperatura de captura y recaptura (gradtemp) según el mes de marcación. Figure 9. Boxplot differences between capture and recapture temperature (gradtemp) according to the tagging month.

\section{Análisis Multivariado}

El dendrograma o cluster con las agrupaciones de los registros de la Tabla 1 al considerar las variables continuas gradiente de temperatura (gradtemp), el tiempo de recaptura, el rumbo, la profundidad y la distancia, indica que aunque pueden establecerse distintos grupos según las diferencias entre ellos (4 a 6), se consideraron 6 para su caracterización (Figura 11). Para el ACP se realizó una primera corrida en la que se estimó la varianza acumulada explicada por cada componente detectada. Las primeras tres componentes explicaron el $86 \%$ de la varianza. En una segunda corrida se descartaron las componentes 4 y 5 . En ese caso, las primeras dos explicaron el $82 \%$. Las variables rumbo y Profundidad resultaron inversamente correlacionadas, esperable según los resultados de la Figura 2. La Profundidad estuvo altamente correlacionada con la distancia, mientras que fue muy baja la correlación entre estas dos últimas variables con el tiempo de recaptura y el gradiente térmico. A mayores rumbos (OSO, 254 ${ }^{\circ}$ promedio), correspondieron menores distancias y profundidades y viceversa. Además, estas tres variables no estarían linealmente vinculadas con el gradiente de temperatura y el tiempo de recaptura. Por lo tanto, las distancias no estarían en relación con "cambios" importantes en las temperaturas de marcado y recaptura, mientras que sí lo estarían con el rumbo y la profundidad.

Para caracterizar los distintos Grupos (1 a 6), se incorporó dicha agrupación como variable categórica al ACP (Figura 12). Los Grupos producto del dendrograma se caracterizaron por:

- G1: altos tiempos de recaptura y gradientes térmicos (registros 1 y 4 ).

- G2: cercanía al lugar de marcación (bajas distancias recorridas) y bajas profundidades, con rumbo mayoritario OSO (registros 2, 6, 7, 8, 9, $10,12,14,19,20,21,22,23,26,27,34,37$ y 39). 
A

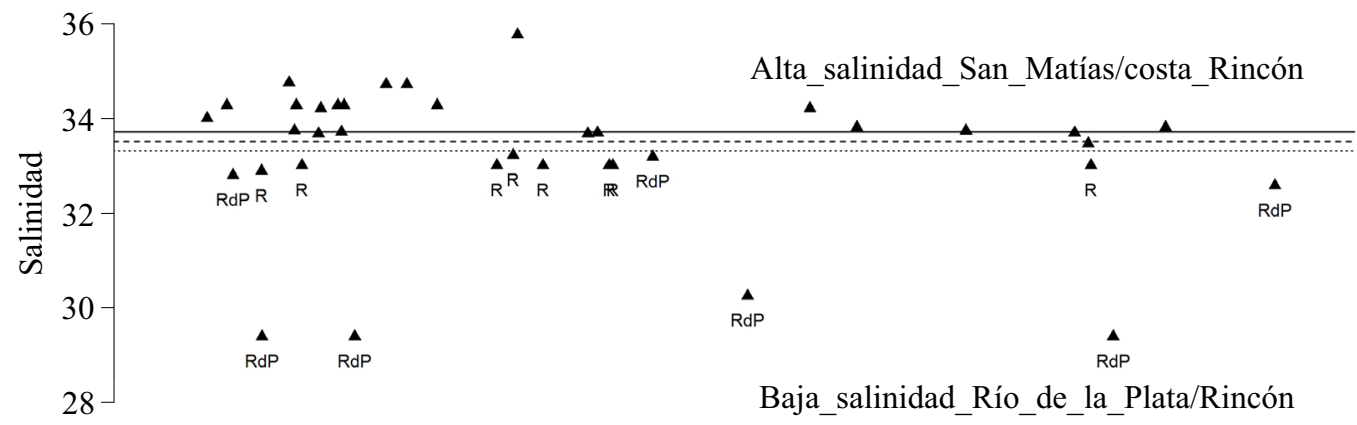

\begin{tabular}{|ccccc|}
\hline 0 & 500 & $1.000 \quad 1.500$ & 2.000 & 2.500 \\
\hline
\end{tabular}

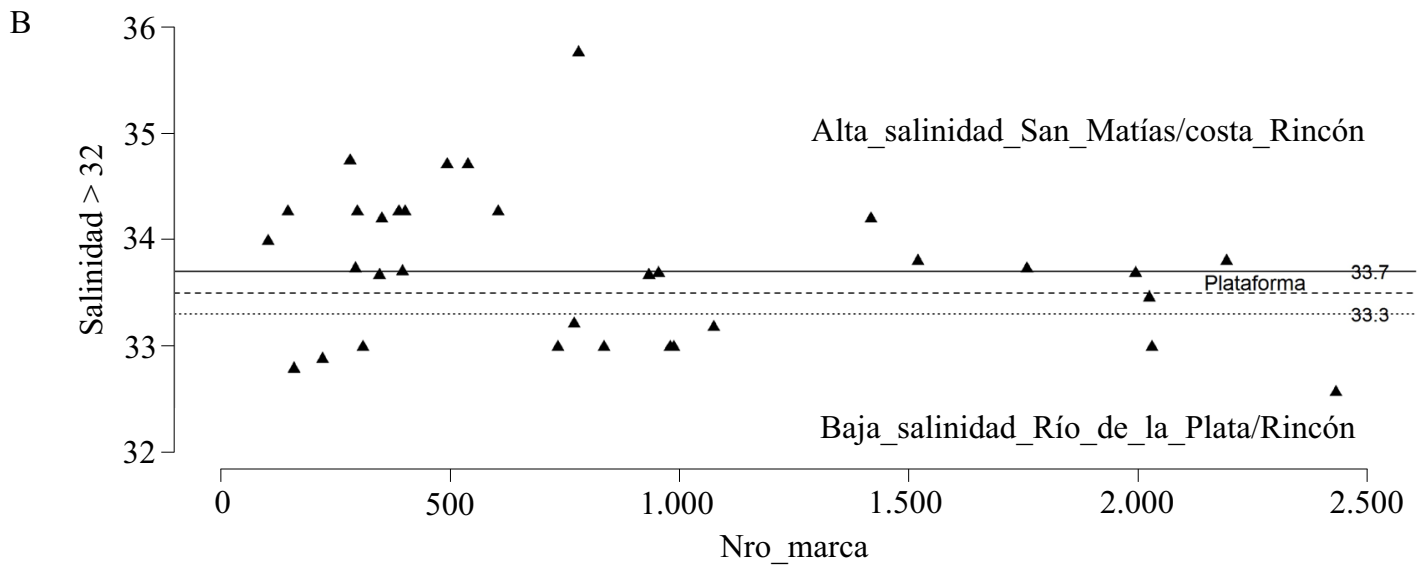

Figura 10. A) Salinidades de superficie promedio mensuales asignadas a cada posición de recaptura según registros históricos de campañas del INIDEP, en una grilla de $0,25^{\circ}$ de Latitud por $0,25^{\circ}$ de Longitud. R: "El Rincón", RdP: Río de la Plata. Línea punteada nivel 33,3 correspondiente a límite de aguas de baja salinidad según Lucas et al. (2005), entre la línea de guiones de 33,5 y la línea llena de 33,7, salinidades superficiales correspondientes con aguas de plataforma, salinidades $>33,7$ aguas de alta salinidad correspondientes con San Matías y área costera de "El Rincón" según los mismos autores. B) Ídem A pero con salinidades superficiales mayores a 32 para visualizar en detalle en este rango.

Figure 10. A) Average monthly surface salinities assigned to each recapture position according to INIDEP's historical records in a grid of $0.25^{\circ}$ Latitude by $0.25^{\circ}$ Longitude. R: "El Rincón", RdP: Río de la Plata. Dotted line level 33.3 corresponding to low salinity water limit according to Lucas et al. (2005), between the dashed line 33.5 and the line full of 33.7, superficial salinities corresponding to the waters of platform, salinities $>33.7$ waters of high salinity corresponding to San Matías and coastal area of "El Rincón" according to the same authors . B) Idem A but with surface salinities greater than 32 to visualize in detail in this range.

- G3: rumbos distintos a los mayoritarios correspondientes con el G2 y G4. Profundidades intermedias, bajo gradiente térmico y distancias de recaptura (registros 11, 17, 18, 31, 32 y 36). Se agruparon en el cluster con el G2 por la cercanía al lugar de marcación y el bajo tiempo de recaptura, pero con rumbo próximos al Norte y al Este. - G4: rumbo ENE, altas distancias de recaptura y profundidades (registros 3, 5, 13, 16, 25, 28, 30, $33,35$ y 40$)$.

- G5: altos tiempos de recaptura, rumbos mayores al promedio OSO y baja profundidad (registros 15, 22 y 24).

- G6: única observación frente al Golfo San Matías. La mayor profundidad registrada $(69 \mathrm{~m}$, registro 29). 


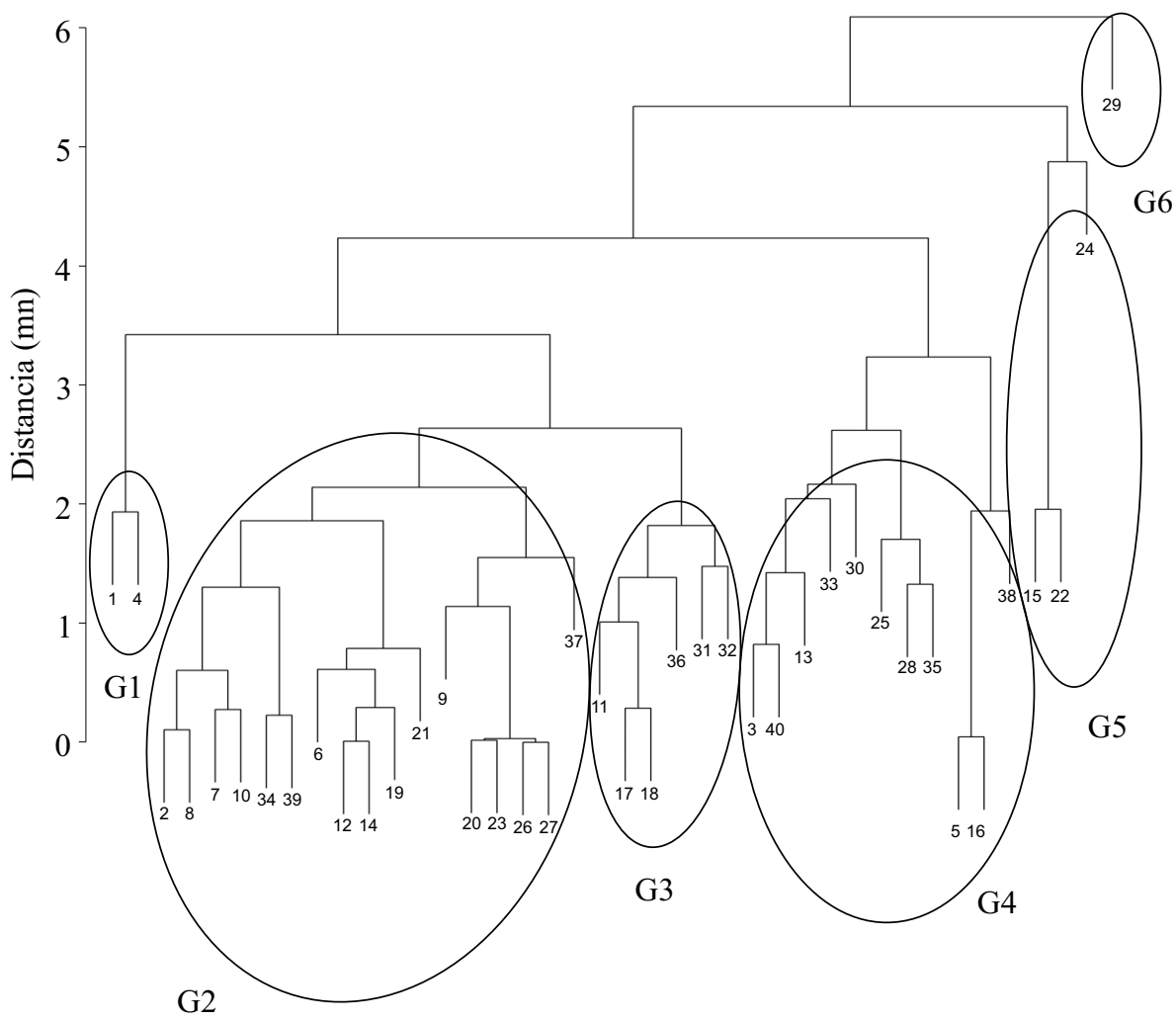

Figura 11. Dendrograma resultado del agrupamiento jerárquico aglomerativo mediante la distancia euclídea y su promedio como enlace, con las variables estandarizadas. El número corresponde a cada registro de la base de datos (Tabla 1). Se señalan los grupos (G1 a G6) caracterizados producto del Análisis de Componentes Principales.

Figure 11. Dendrogram from the agglomerative hierarchical clustering by euclidean distance and its average as a link with standardized variables. The number corresponds to each record in the database (Table 1). The groups (G1 to G6) characterized by the Principal Component Analysis are indicated.

De manera similar se realizó el mismo tipo de análisis considerando como variable categórica el mes de marcación (Figura 13). No habría tendencia clara de alguna agrupación en relación con el momento del marcado, hecho esperable según la Figura 2. Lo mismo resultó al evaluar el sexo, por lo tanto no habría tendencia clara de agrupación en relación con el sexo (Figura 14). Además, según el test de Chi cuadrado, la proporción de sexos de los individuos recapturados no resultó significativamente distinta a 1:1 $(\mathrm{p}=0,53)$.

Los resultados del MLG, construído para evaluar estadísticamente que variables disponibles (continuas y discretas) podrían aportar a explicar la variación de la distancia de recaptura, indica- ron que el ajuste no fue bueno, aunque se cumplieron los supuestos de normalidad de residuales y homocedasticidad (Figura 15). Este modelo resultó apenas significativo para explicar la variabilidad de la distancia:

Error estándar Residual: 1,166 con 26 GL Multiple $\mathrm{R}^{\wedge} 2$ : 0,5355, Ajustado $\mathrm{R}^{\wedge} 2: 0,3033$ F-estadístico: 2,306 con 13 y $26 \mathrm{GL}, \mathrm{p}=0,03381$

En la tabla de ANOVA correspondiente, se observa la falta de significación de las variables explicativas consideradas, salvo para la variable mes_marcado que resultó apenas por debajo del $5 \%$ de probabilidad (Tabla 3 ). 


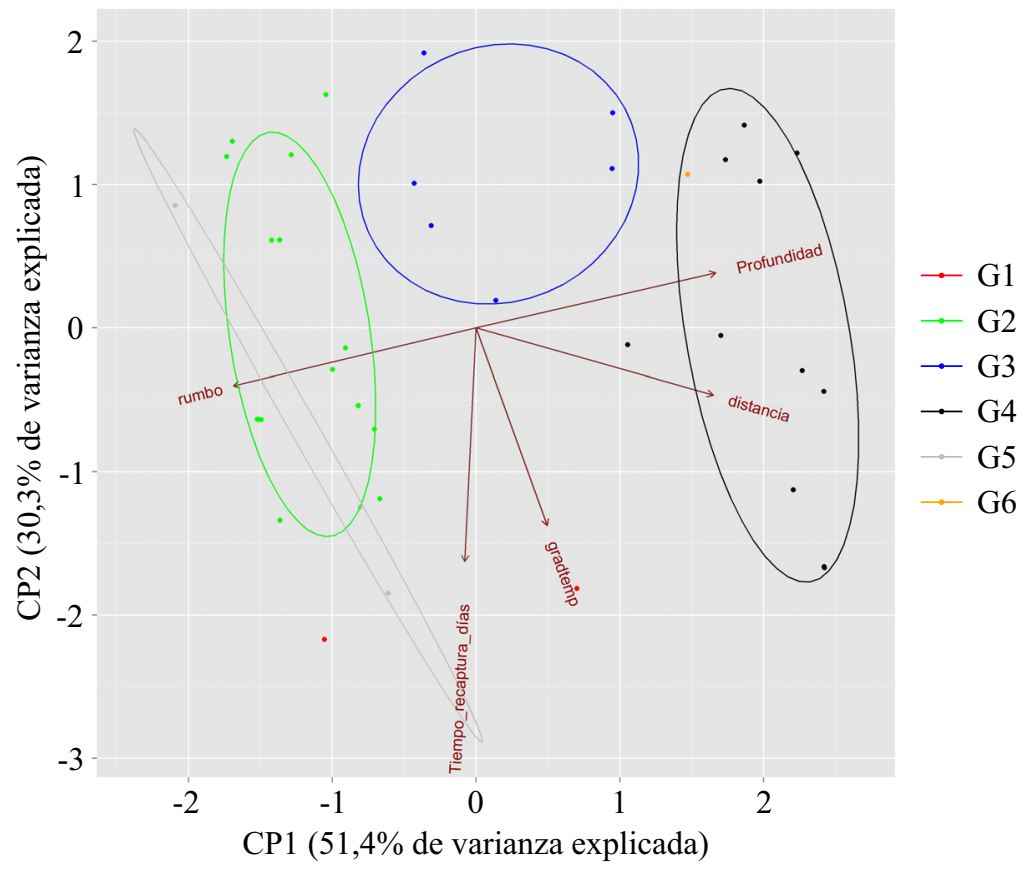

Figura 12. Cada color corresponde con las agrupaciones producto del ACP, señaladas en las leyendas (ver texto) y los diferentes contornos.

Figure 12. Each color corresponds with the groupings product of the ACP, identified in the legends (see text) and the different contours.

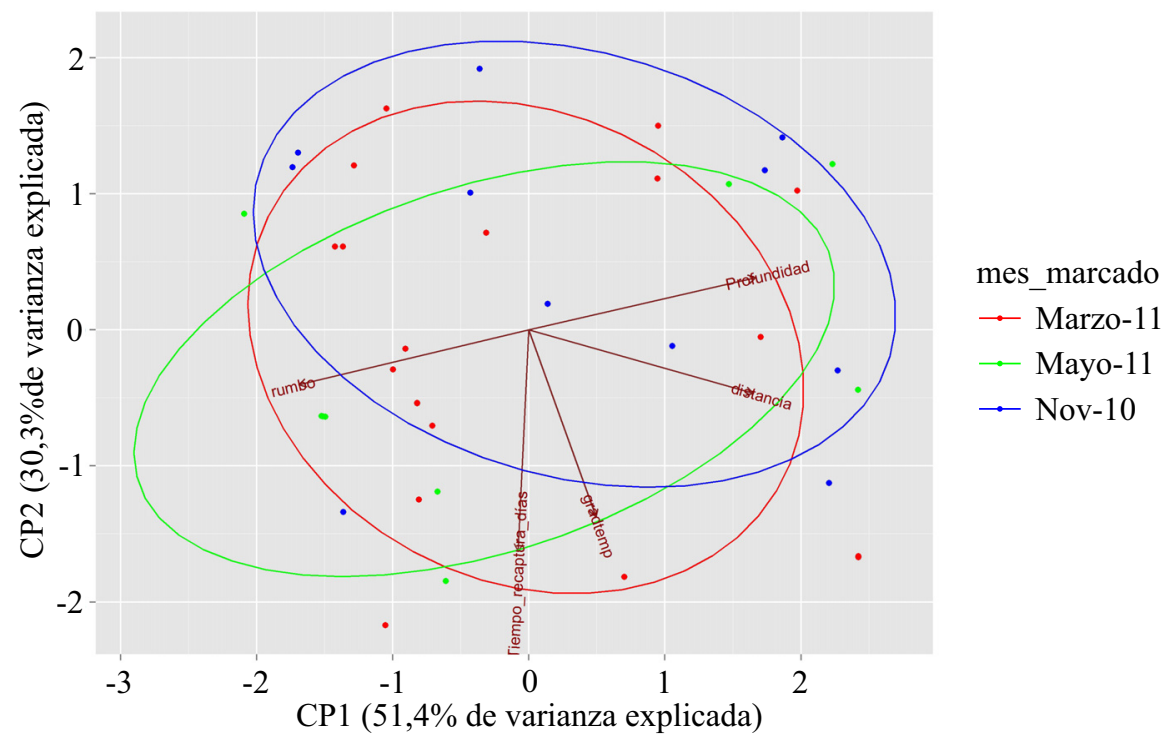

Figura 13. Cada color de los puntos se corresponde con el mes de marcación (mes_marcado) y los diferentes contornos para cada uno de ellos producto del ACP.

Figure 13. Each dots color corresponds to the tagging month (mes_marcado) and the different contours for each of them product of the $A C P$. 


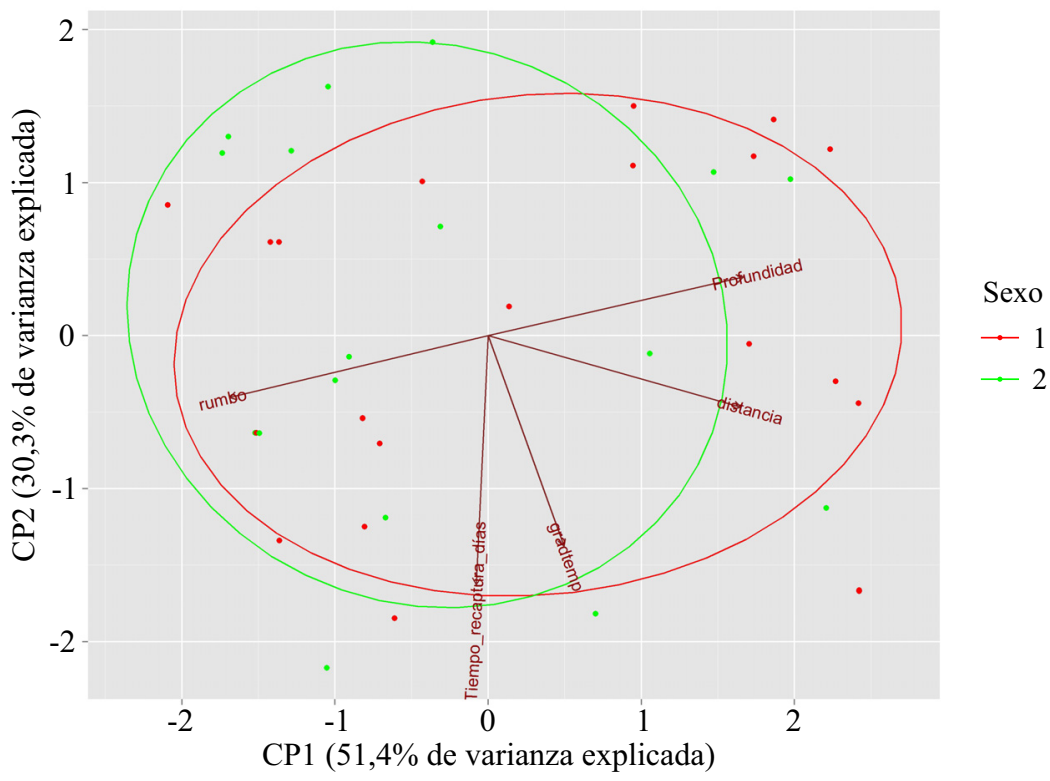

Figura 14. Cada color de los puntos se corresponde con las agrupaciones para cada sexo (número 1 machos en rojo y 2 hembras en verde) y los diferentes contornos para cada uno de ellos producto del ACP.

Figure 14. Each color of the dot corresponds to the groupings for each sex (number 1 males in red and 2 females in green) and the different contours for each of them product of the ACP.

A

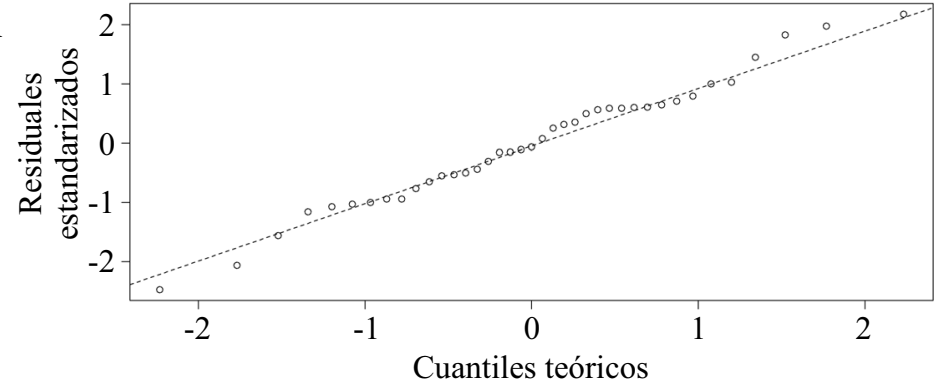

B

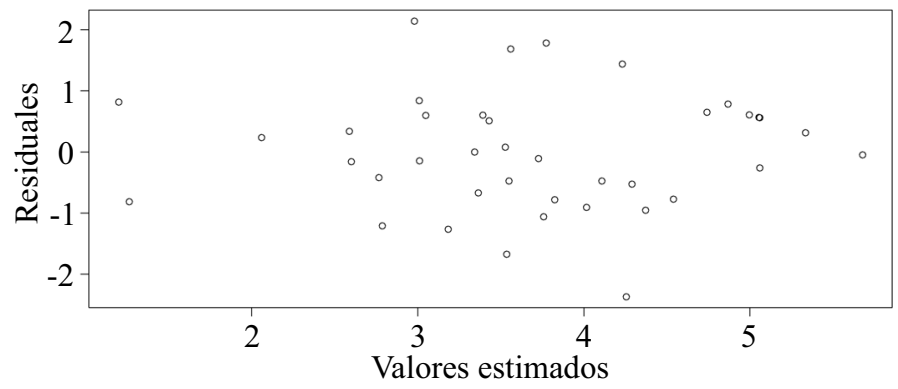

Figura 15. Gráficos de diagnóstico del ajuste del MLG lognormal con variable respuesta el logdistancia (mn) entre las posiciones asignadas de marcado y recaptura. A) Gráfico QQ de residuales estandarizados. B) Residuales crudos versus valores estimados.

Figura 15. Diagnostic graphs of the Lognormal MLG with response variable logdistancia (mn) between the assigned taggingrecapture positions. A) Residuals standardized $Q Q$ plot. B) Raw residual versus estimated values. 
Tabla 3. Tabla de ANOVA correspondiente al GLM lognormal con el logdistancia como variable respuesta. Tabla 3. ANOVA corresponding to the lognormal GLM with logdistance as a response variable.

\begin{tabular}{lcccc}
\hline & GL & Suma de cuadrados & F & $\operatorname{Pr}(>\mathrm{F})$ \\
\hline $\log$ (talla_cap_cm) & 1 & 0,5820 & 0,4284 & 0,5185 \\
sexo & 1 & 4,1168 & 3,0303 & 0,0935 \\
gradtemp & 1 & 4,6777 & 3,4432 & 0,0749 \\
log(Tiempo_recaptura_días) & 1 & 0,2699 & 0,1987 & 0,6595 \\
mes_marcado & 2 & 9,1785 & 3,3781 & $0,0496^{*}$ \\
mes_recaptura & 7 & 21,9015 & 2,3031 & 0,0574 \\
Residual & 26 & 35,3219 & & \\
\hline
\end{tabular}

\section{Desplazamientos}

En la Figura 16 se presentan señaladas con flechas, a modo de esquema según el mes de marcación, las potenciales trayectorias de los individuos entre las posiciones de marcado y de recaptura correspondientes, en las que se detallan el tiempo de permanencia en libertad de cada individuo. En la Figura 16 B, que corresponde a las marcaciones realizadas en el mes de marzo de 2011, no se presentan las flechas con rumbos OSO debido al alto número de recapturas cercanas a Monte Hermoso.

Los resultados mencionados hasta aquí, indicaron una tendencia a que un grupo de individuos se desplace a zonas lejanas, con rumbo ENE, mientras que otro parece hacerlo a aguas cercanas con rumbo OSO, aunque existen otras situaciones como la recuperada en la cercanía del Golfo San Matías, que indicarían también desplazamientos en otras direcciones. Los resultados del test espacial de Rao (1967) al considerar todos los datos en conjunto, no permiten rechazar la hipótesis nula de uniformidad en las direcciones de desplazamiento desde el lugar de marcación. El mismo test se aplicó separando los datos por cada uno de los meses de marcación, con los mismos resultados. Pudo observarse que hubo individuos recapturados tanto en la Zona Norte como cerca de Monte Hermoso con tiempos similares de perma- nencias en libertad, correspondientes a los tres meses de marcación. Esto podría indicar que, principalmente para los individuos que presentaron los menores tiempos de recaptura, algunos migraron al Norte y otros permanecieron en el área de Monte Hermoso. También hubo recapturas en ambas zonas para tiempos en libertad mayores a 6 meses (Figura 16). A medida que incrementa el tiempo en libertad, aumenta la incertidumbre sobre las trayectorias, ya que los individuos pudieron realizar desplazamientos en otras direcciones o haber realizado numerosos en sentido ENE y retornar.

\section{Tiempos de permanencia en áreas de interés particulares. Zona Común de Pesca Argenti- no-Uruguaya}

La proporción y el tiempo en libertad de los ejemplares cuya recaptura se produjo en rumbos menores a $122^{\circ}$, dentro y fuera de la ZCPAU, indica que once individuos permanecieron alrededor de un $60 \%$ de su período en libertad en la ZCPAU o en aguas próximas de jurisdicción provincial, mientras que los 7 restantes permanecieron el 100\% fuera de la ZCPAU (Figura 17). Si se considera el mismo criterio, para los ejemplares restantes recapturados en posiciones correspondientes a rumbos mayores a $122^{\circ}$ (22 individuos, Tabla 1), el $100 \%$ de su tiempo en libertad trans- 
currió fuera de la ZCPAU. Por lo tanto, más de un $70 \%$ de los individuos recapturados pasaron el $100 \%$ de su tiempo en libertad fuera de la ZCPAU, mientras que los restantes permanecieron alrededor de un $60 \%$ dentro.

\section{DISCUSIÓN}

\section{Relaciones entre los desplazamientos horizon- tales del gatuzo (Mustelus schmitti) en el ecosis- tema costero bonaerense y su ciclo de vida}

Posiciones de recaptura y relación con factores ambientales

Según los resultados del ACP y del GLM, ninguna de las variables consideradas fue altamente significativa para explicar la variación de la distancia recorrida. Existió un conjunto de individuos que se desplazó y fue recapturado en zonas distantes, al sur de la Bahía de Samborombón entre $37^{\circ} \mathrm{S}$ y $38^{\circ} \mathrm{S}$ al este de la costa de la Provincia de Buenos Aires, y en proximidades del área de descarga del Río de la Plata (Zona Norte). Otro grupo mayoritario parece haber permanecido o vuelto a la zona de "El Rincón" (Zona Sur).

La relación de las concentraciones de condrictios con áreas frontales ya fue mencionada por Lucífora et al. (2012), particularmente vinculadas con ambos frentes ya descriptos. Estos autores señalaron que algunas rayas costeras y el pez ángel, son más abundantes y tienen zonas de cría a lo largo de las áreas frontales "estuarinas". Según los resultados de la marcación, los RE en los que se declaró la mayor proporción de recapturas, tanto en la Zona Norte (RE 3655 y 3755) como en "El Rincón" (RE 3961 y 3960), fueron coincidentes con las áreas de mayor actividad de la flota comercial (Massa et al. 2004, Hozbor y Massa, 2015) en proximidades de las zonas frontales. Los casos restantes, con la salvedad del caso del procedente de la boca del Golfo San Matías, pueden considerarse como posiciones de "transi- ción" entre ambas. En la franja costera entre Necochea y Mar Chiquita, tanto en otoño como en primavera, se encontraron individuos adultos de tallas intermedias, a diferencia de las distribuciones de tallas tanto de la Zona Norte como de "El Rincón", donde se detectaron tanto juveniles como adultos (Cousseau 1986; Massa et al. 2004).

Recientemente ha sido señalada nuevamente la importancia ecológica de los frentes del Atlántico Sudoccidental (Acha et al. 2015), en particular en el caso de la megafauna como tortugas, aves y mamíferos marinos (González Carman et al. 2016). La ubicación de las áreas frontales tanto costeras como oceánicas del litoral de la Provincia de Buenos Aires, señaladas por González Carman et al. (2016), y el total de las posiciones de las recapturas de gatuzo, evidencian la asociación espacial de las recapturas con los frentes tanto del Río de la Plata como de "El Rincón" (Figura 18). Ya fue señalada la limitante del uso de marcas convencionales respecto del sesgo vinculado a la distribución espacial del esfuerzo pesquero. Sin embargo, fue coincidente la distribución espacial del esfuerzo y en consecuencia de las capturas de gatuzo, con aquellos RE cercanos a las áreas frontales.

\section{Desplazamiento de juveniles}

Cousseau (1986) y Cousseau et al. (1998) señalaron la presencia de dos áreas de cría, una al sur de Bahía Samborombón frente a Cabo San Antonio $\left(36^{\circ} 20^{\prime} \mathrm{S}\right)$ y otra en "El Rincón" desde Claromecó hasta Faro Segunda Barranca, ambas coincidentes con las áreas frontales señaladas. Massa et al. (2004) también asociaron ambos sistemas frontales de salinidad de fondo con las áreas de cría de gatuzo. Cortés et al. (2011), mediante datos de campañas de investigación, indicaron que los juveniles de gatuzo $(<50 \mathrm{~cm}$ de talla) permanecerían en la costa durante el año, en profundidades menores a $25 \mathrm{~m}$. También mencionaron dos áreas de cría, una cercana a Punta Medanos en la región costera y otra en la parte 

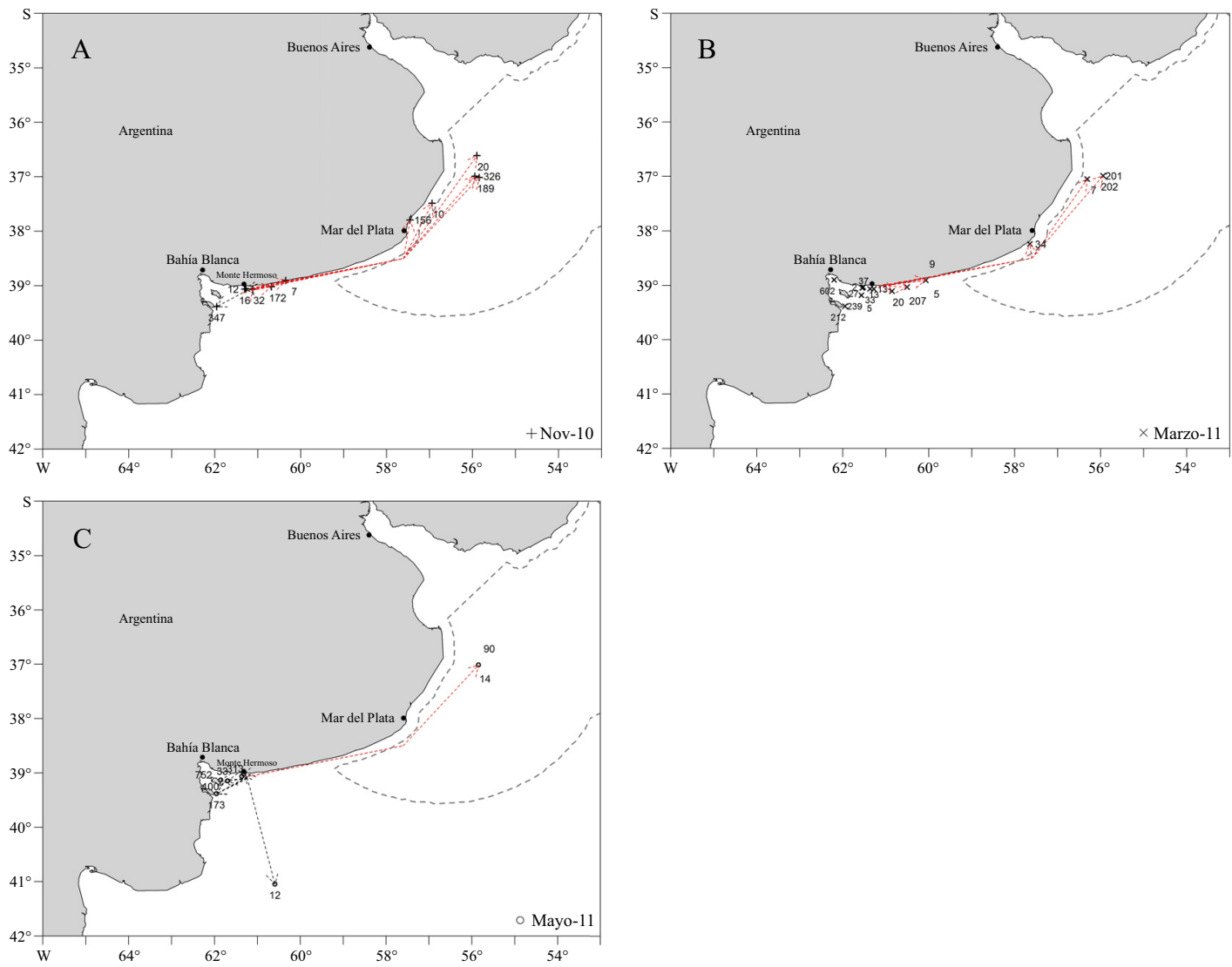

Figura 16. Esquema de las trayectorias de los individuos marcados en noviembre de 2010 (A), marzo (B) y mayo de 2011 (C). En la posición de recaptura se presenta el tiempo de permanencia en el mar. En líneas punteadas y flechas las trayectorias considerando los rumbos mayoritarios ENE y OSO.

Figure 16. Trajectories scheme of each individuals tagging in November 2010 (A), March (B) and May 2011 (C). In the recapture position is presented the time at liberty. Dotted lines and arrows indicate trajectories considering the main courses ENE and WSW.

interna de "El Rincón". También se señaló que tanto el área de influencia del frente salino del Río de la Plata como aquel de "El Rincón" (Figura 18), desde el punto de vista de utilización del hábitat, cumplen un papel importante como áreas de cría, ya que los juveniles dispondrían de alimento abundante y altas temperaturas relativas que contribuiría a su crecimiento rápido (Oddone et al. 2007; Cortés et al. 2011). Estas áreas frontales favorecerían la retención de larvas de especies bentónicas y por lo tanto el establecimiento de invertebrados bentónicos como fuente de alimento (Acha et al. 2015).
Algunos individuos de alrededor de $50 \mathrm{~cm}$ de talla al momento de la marcación fueron recapturados a grandes distancias relativas. Según los antecedentes, la talla de primera madurez para las hembras fue estimada entre 56 y $72 \mathrm{~cm}$, y para los machos entre 55 y $60 \mathrm{~cm}$ (Colautti et al. 2010). Los ejemplares correspondientes a las marcas 953 y 1073, machos marcados en mayo de 2011, de 52 y $48 \mathrm{~cm}$ de talla respectivamente, fueron recuperados al norte de Mar del Plata a los 14 y 90 días. Ambos individuos, según la escala de madurez macroscópica (estadio 2, Colonello et al. 2007), se clasificaron como juveniles al momento de la mar- 


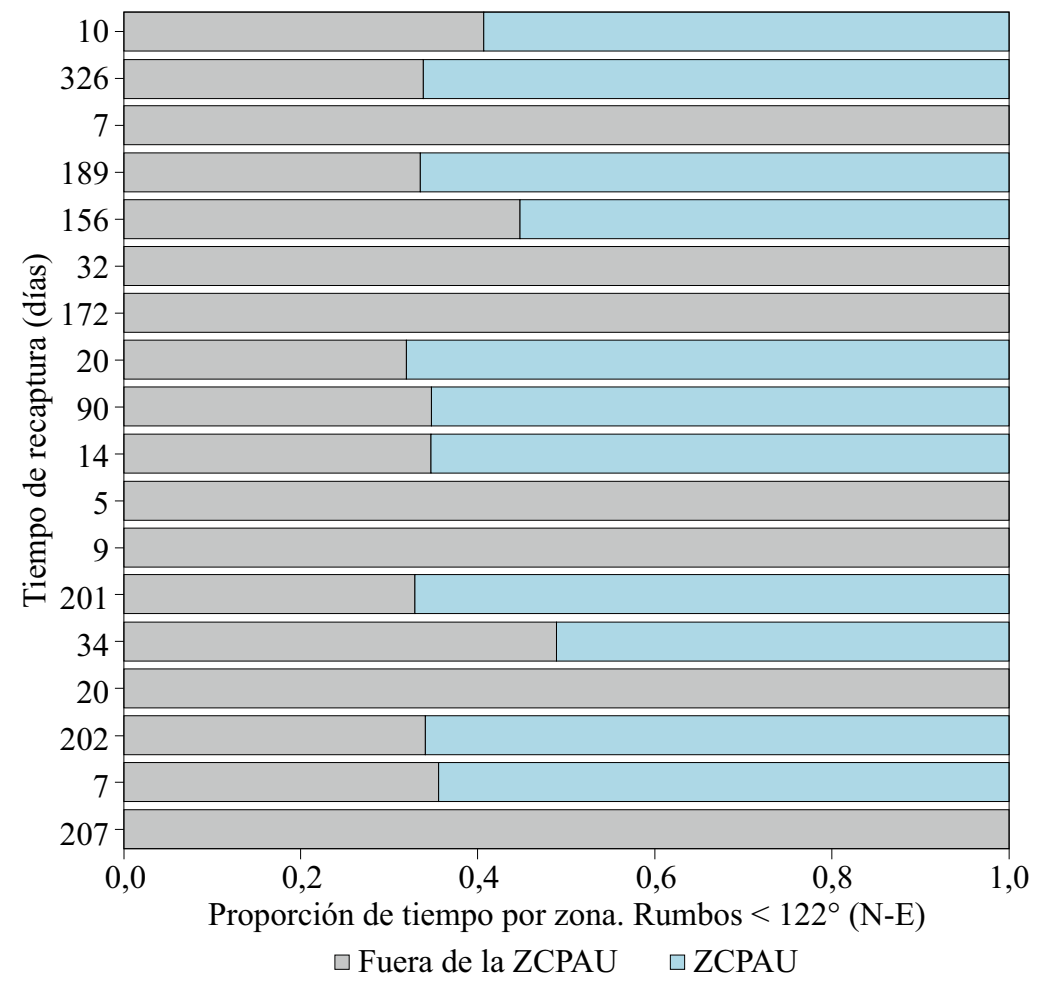

Figura 17. Proporción de tiempo en libertad dentro y fuera de la Zona Común de Pesca Argentino-Uruguaya (ZCPAU). Cada barra de distinto color representa el número de días transcurrido en cada zona correspondiente a los 18 individuos recapturados con rumbos menores a $122^{\circ}$ a partir de las posiciones de marcado.

Figure 17. Proportion of time at liberty inside and outside the Argentine-Uruguayan Common Fishing Zone (ZCPAU). Each bar of different color represents the number of days spent in each zone by the 18 individuals recaptured with courses less than $122^{\circ}$ from the tagging positions.

cación. Esto indicaría desplazamientos de individuos en sentido sur-norte entre ambas zonas frontales que podrían ser considerados juveniles. Los antecedentes sobre crecimiento (Hozbor et al. 2010) sugieren para las edades correspondientes a juveniles (edades 1 y 2) un incremento promedio de alrededor de $5 \mathrm{~cm}$ año-1 en ambos sexos. Por lo tanto, aunque no se dispuso de las tallas al momento de la recaptura de los individuos mencionados, es improbable que hayan alcanzado la madurez sexual en esos plazos.

\section{Hipótesis sobre los desplazamientos de adultos}

Al analizar los resultados de dos campañas de investigación, una realizada en primavera y otra en otoño, Cousseau (1986) señaló que habría un movimiento con fines reproductivos en primavera desde aguas más profundas hacia aguas más costeras, evidenciado tanto por las abundancias como por la estructura de tallas. Los adultos se concentran en la costa en la primavera tardía para la parición y aparearse tanto al norte como en "El Rincón", luego migran hacia mayores profundidades (Cousseau 1986; Massa et al. 2004). Cortés et al. (2011) mencionaron que las abundancias de los adultos estarían asociadas desde el punto de vista ambiental, con la profundidad y la temperatura. En otoño-invierno la abundancia de adultos estaría vinculada con aguas más profundas $(>25 \mathrm{~m})$, mientras que en primavera, en la temporada reproductiva, los adultos migrarían hacia la costa segre- 
A

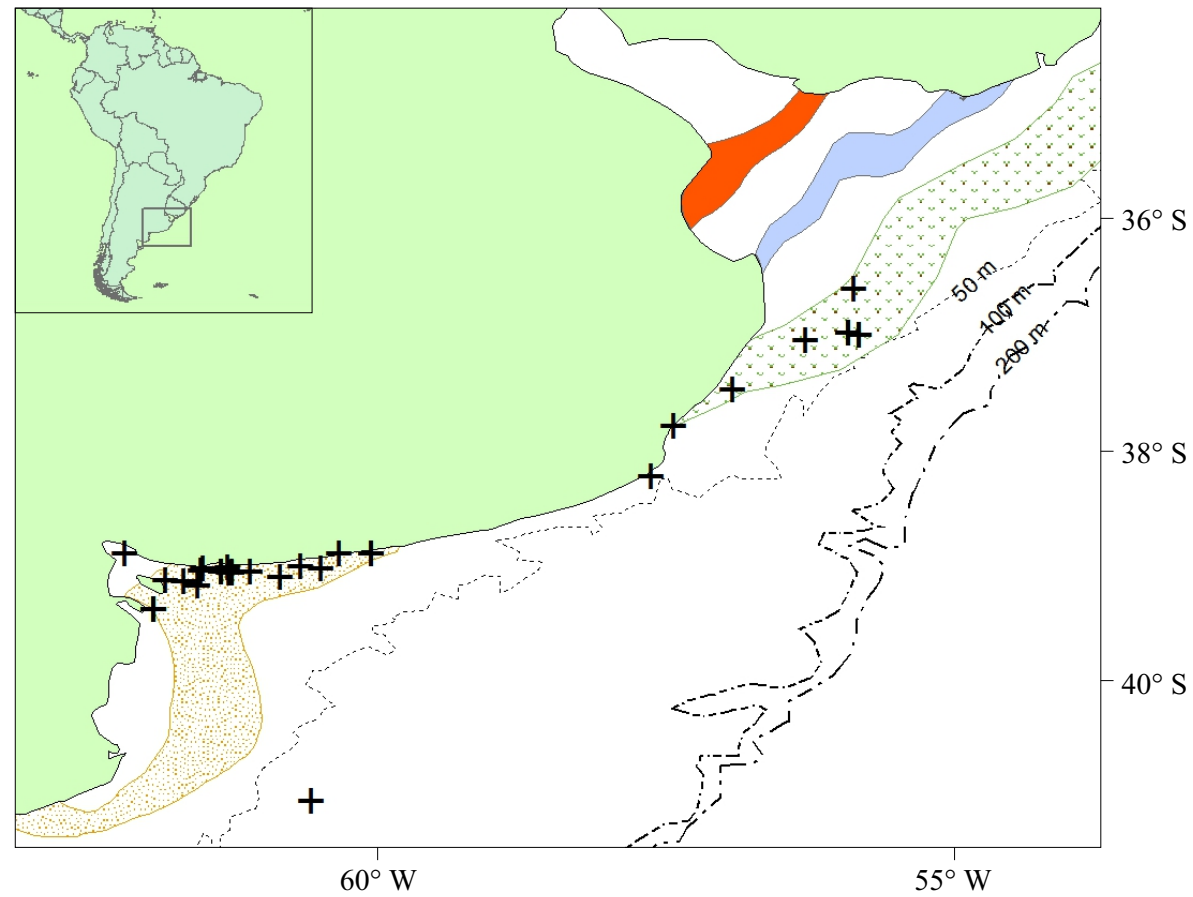

B

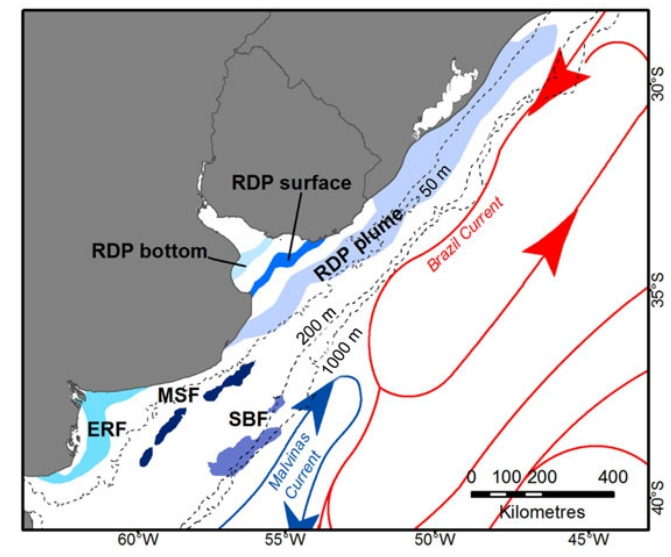

+ Posición de recaptura

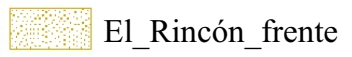

RdP_frente_pluma

RdP_frente_superficie

RdP_frente_fondo

Isobatas

$50 \mathrm{~m}$

--.--.. $100 \mathrm{~m}$

$-\cdot-\cdot 200 \mathrm{~m}$

$\begin{array}{lllll}0 & 1530 & 60 & 90 & 120\end{array}$

Millas náuticas

Figura 18. A) Posiciones de recapturas de gatuzo con la proyección de las áreas frontales en la costa de la Provincia de Buenos Aires extraídas de González Carman et al. (2016) y cedida por los autores. Frentes salinos del Río de la Plata y "El Rincón”. B) Posición de los frentes en el litoral de la Provincia de Buenos Aires y aguas de la plataforma continental tomada de González Carman et al. (2016): RDP bottom frente de fondo del Río de la Plata, RDP surface frente de superficie del Río de la Plata, RDP plume frente de la pluma del Río de la Plata, ERF frente de "El Rincón”, SBF frente del talud de la plataforma continental, MSF frente de la plataforma continental media.

Figure 18. A) Recapture positions with the projection of the Buenos Aires Province frontal areas extracted from González Carman et al. (2016) and ceded by the authors. Rio de la Plata and "El Rincón" saline fronts. B) Position of the fronts on the coast of Buenos Aires Province and waters of the continental shelf taken from González Carman et al. (2016). RDP bottom: Río de la Plata bottom front, RDP surface: Río de la Plata surface front, RDP plume: Río de la Plata plume front, ERF: "El Rincon" front, SBF: continental shelf break front, MSF: mid-continental shelf front. 
gados sexualmente. Colautti et al. (2010) indicaron máximas abundancias relativas de hembras con embriones y machos fluyentes dentro de Bahía Anegada en primavera, al sur de "El Rincón", por lo que habría movimiento hacia adentro de la Bahía en primavera temprana y saldrían en primavera tardía y verano, estando ausentes en otoño e invierno. Esta Bahía representaría un área de nacimiento y cría de juveniles.

Oddone et al. (2007), mediante datos de campañas de investigación realizadas en la ZCPAU, concluyeron que los adultos de ambos sexos preferirían aguas más costeras y cálidas durante la primavera y el verano, con fines reproductivos. Pereyra et al. (2008) con datos de observadores a bordo de la flota comercial uruguaya, señalaron que en la plataforma del sector uruguayo de la ZCPAU, las diferencias entre las distribuciones de tallas por sexo a menores y mayores profundidades de $50 \mathrm{~m}$, estarían en relación con los movimientos reproductivos. La mayor proporción de hembras maduras en aguas de menor profundidad en primavera y verano indicaría la temporada de reproducción para esta zona. Luego de ser fecundadas, las hembras se desplazarían nuevamente hacia aguas más profundas.

La marcación de ejemplares en noviembre correspondió a la temporada reproductiva de la especie (Menni et al. 1986; Sidders et al. 2005; Cortés et al. 2011, entre otros). En particular, existieron dos casos, los ejemplares identificados con las marcas 2026 y 2074 . La hembra con la marca 2074 fue recapturada 11 meses después en la Zona Norte y el ejemplar restante (macho, marca 2026) casi un año después en las islas frente a Monte Hermoso. Los tiempos de las recapturas eran próximos a la siguiente temporada reproductiva, pero estos individuos se encontraron en zonas distantes entre sí. En el caso correspondiente a la hembra con la marca 2074, pudo recuperarse el ejemplar entero y se verificó que, con $64 \mathrm{~cm}$ de talla al momento de la recaptura, asignada al 11 de octubre de 2011, estaba grávida con embriones de $22 \mathrm{~cm}$ de longitud de longitud total modal. Aunque la talla observada de los embriones pueda ser considerada dentro de su variabilidad al momento de la parición (Sidders et al. 2005; Cortés 2007), no se puede afirmar que estuviera próxima a parir en la Zona Norte. Menni et al. (1986) señalaron que la talla (longitud total) de los embriones al momento de la parición sería de $27 / 28 \mathrm{~cm}$, mientras que para Oddone et al. (2005) sería de $26 \mathrm{~cm}$. Esto podría indicar que esta hembra aún no se encontraba en esta situación. El ejemplar macho restante (Marca 2026) no pudo ser recuperado, pero correspondía a un individuo maduro sexualmente, de $68 \mathrm{~cm}$ de talla en el momento de la marcación, con fecha de recaptura asignada al 1 de noviembre de 2011. Además, existieron recapturas en la Zona Norte a los 10 y 20 días luego de la marcación en Monte Hermoso en noviembre de 2010, por lo que fue posible para otros ejemplares marcados en el mismo mes, realizar ese trayecto en tiempos que permitirían su retorno al lugar de marcación. Sin embargo, la presencia de recapturas en la Zona Norte, la falta de correlación detectada entre las variables distancia, mes de marcación y tiempo de recaptura, además del desplazamiento sur-norte de los juveniles ya planteado, podría indicar un importante grado de mezcla de juveniles y reproductores con baja filopatría, definida como el retorno preferencial de reproductores a sus sitios de nacimiento y por lo tanto un uso multigeneracional que llevaría al desarrollo de cierta estructura poblacional desde el punto de vista genético (Chapman et al. 2015). Habría evidencias en el caso del gatuzo de que alguna proporción importante de ejemplares podrían moverse a lo largo del litoral y, en condiciones propicias, se apareen y realicen la parición en distintos lugares a lo largo de su vida.

Los antecedentes sobre el gatuzo mencionados hasta aquí, con evidencias producto de distintas fuentes de información, indican un desplazamiento de ambos sexos hacia aguas costeras con agregación con fines reproductivos en primavera, tanto en la Zona Norte como en la Sur, en proximidades de ambas zonas frontales. Los resultados 
de la marcación no sugieren una agregación por sexo ni por mes de recaptura. Tampoco hubo recapturas en profundidades mayores a $50 \mathrm{~m}$ (salvo el caso frente al Golfo San Matías), lo que indicara una tendencia a que algunas tallas o sexos se desplazaran a mayores profundidades en alguna época del año. Esto pudo no ser percibido debido al número de recapturas y/o de la distribución del esfuerzo de la flota que operó a profundidades mayores de $50 \mathrm{~m}$. Sin embargo, aparecieron evidencias de desplazamientos que tendrían rumbo ENE a partir del lugar de marcación, de una proporción importante de individuos de ambos sexos en un amplio rango de tallas (juveniles y adultos). Estos desplazamientos se realizarían en escalas temporales diversas, aún en plazos muy cortos debido a las velocidades de natación detectadas (hasta $19 \mathrm{~km}$ día $^{-1}$ ). Hubo recapturas en la Zona Norte a $\operatorname{los} 7,10$ y 14 días a partir del momento de marcación, en los tres meses (noviembre, marzo y mayo) en que se marcaron individuos en Monte Hermoso. Además, hubo recapturas de individuos de ambos sexos (macho juvenil de $48 \mathrm{~cm}$ y hembra adulta de $63 \mathrm{~cm}$ ) en invierno (agosto), en proximidades del lugar de marcación luego de 90 y 100 días de permanencia en libertad, lo que indicaría que tanto juveniles como adultos permanecerían o potencialmente "volverían" a la Zona Sur. Estos resultados sugieren una dispersión a partir de la zona de marcación, principalmente en sentido ENE, pero también en otras direcciones por ejemplo el caso frente al Golfo San Matías.

Desde el lugar de marcación, no se detectaron indicios de desplazamientos de juveniles en primavera ni de los machos adultos mayoritariamente en alguna temporada en sentido ENE. Tampoco de los adultos de ambos sexos principalmente en invierno con este mismo rumbo de desplazamiento (Oddone et al. 2005, 2007). No se declararon recapturas al norte de $36^{\circ} \mathrm{S}$, por lo tanto no puede inferirse sobre posibles desplazamientos hacia menores latitudes.

Es probable que haya permanencias en la zona de marcación durante todo el año, aunque no es posible caracterizarlos como residentes debido al desconocimiento de sus trayectorias. Esto no excluiría las concentraciones reproductivas detectadas con numerosos antecedentes y los desplazamientos en sentido oeste-este, o de mayores a menores profundidades tanto en la Zona Norte como en la Sur. Pero, según los resultados de la marcación, existiría un importante grado de "mezcla" en sentido sur-norte de adultos reproductores de ambos sexos y juveniles, hecho congruente con los antecedentes de genética de poblaciones (Pereyra et al. 2010; Andreoli y Trucco 2015,2018 ) y con el test de uniformidad en las direcciones de dispersión, desde el lugar de marcación para los distintos meses en que se realizó la marcación. Por lo tanto, habría fidelidad por sitios de reproducción pero sin reconocimiento por parte de los reproductores de su propio sitio de nacimiento (filopatría).

Existen antecedentes sobre el uso de bahías y estuarios costeros para alimentación, apareamiento y parición, como en el caso de $M$. californicus y $M$. henlei en la costa de California (Espinoza et al. 2011). En el caso de M. californicus, mediante marcas acústicas a lo largo de dos años, se observó el uso de una bahía costera por parte de individuos de esta especie. De 22 ejemplares marcados solo dos tuvieron una permanencia superior a dos meses y ninguno regresó con posterioridad luego de su salida durante el invierno. Su abundancia dentro de la bahía estaría relacionada con mayores temperaturas en la temporada cálida y cambios en la disponibilidad de presas. Este antecedente sería un indicador similar al gatuzo en cuanto a la baja "fidelidad" por algunos ambientes particulares. Contrariamente en M. mustelus en Sudáfrica, también mediante el uso de marcas acústicas y durante dos años, habría evidencias de fidelidad y residencia en una bahía costera al evaluar el uso de un área marina protegida delimitada dentro de ella. Sin embargo, el análisis se realizó solo sobre los individuos presentes durante por lo menos un ciclo anual en la bahía, 15 sobre 24 marcados (da Silva et al. 2013). 
Mediante el uso de marcas convencionales, recientemente se propuso a $M$. asterias en el Atlántico Nororiental como especie filopátrica, debido a variaciones estacionales de las recapturas por zonas, pero sin considerar las variaciones correspondientes al esfuerzo pesquero en dichas zonas (Brevé et al. 2016). La carencia de información sobre la distribución espacio temporal del esfuerzo influye en las consideraciones respecto de las definiciones de residencia, fidelidad por un sitio y filopatría, al utilizar marcas convencionales. La componente genética debe ser considerada para la calificación de una especie como filopátrica (Chapman et al. 2015).

Según Schlaff et al. (2014), en rayas y tiburones los vínculos entre los movimientos y el medio ambiente están correlacionados, y no proveen evidencia concluyente de que un único factor abiótico o biótico, sea la causa de los movimientos. Además, estos factores no ocurren separadamente, por lo tanto se dificulta determinar cual es de mayor importancia o cuando existen interacciones entre ellos. Los movimientos estarían conducidos por una combinación de factores, bióticos y abióticos, con una selección de hábitat resultante de un balance entre la necesidad de optimizar funciones metabólicas y fisiológicas, y a la vez acceder a recursos valiosos, por ejemplo alimento o refugio. Espinoza et al. (2011) señalan para M. californicus que los patrones espaciales de abundancia estarían influenciados por la temperatura y la disponibilidad de presas. En el caso de M. henlei en California, se movería hacia aguas someras en condiciones de mareas entrantes a una bahía costera, presumiblemente con fines alimenticios (Campos et al. 2009). Elisio et al. (2015) propusieron a la temperatura como disparador abiótico de la agregación reproductiva de $M$. schmitti en la zona de "El Rincón". Jaureguizar et al. (2016), analizando campañas de investigación en "El Rincón" en la temporada reproductiva, indicaron vínculos entre la abundancia y factores abióticos como profundidad, cercanía a la costa, salinidad y la descarga de los ríos próximos. Según los resul- tados de este trabajo, mediante el método de marcado y recaptura, la componente biótica parece relevante. Como ya fue discutido, hubo casos de ejemplares adultos marcados en noviembre de 2010 (temporada reproductiva) en Monte Hermoso, que fueron recapturados al norte a más de 400 $\mathrm{km}$ de distancia luego de 10 y 20 días de libertad. Dicha componente asociada con áreas frontales estaría vinculada con la disponibilidad de recursos alimenticios. La descripción de la dieta de la especie (Bellegia et al. 2012) parece coincidir con las características propias de las áreas frontales señaladas por Acha et al. (2004), ya que ambas zonas "estuarinas" se caracterizan por fondos blandos, habitados principalmente por organismos bentónicos como poliquetos, bivalvos y crustáceos. También serían áreas de depredación para especies de alto nivel trófico, como tiburones costeros y rayas. Lucífora et al. (2012) propusieron que la gran diversidad de especies de condrictios en ambas zonas frontales sería producto de la abundancia de presas (invertebrados bentónicos). Estos indicios, en conjunto con las recapturas de gatuzos adultos y juveniles, indicaría la utilización de ambas zonas frontales a lo largo del año por parte de esta especie.

Francis (1988) estableció objetivos similares a este trabajo mediante el uso de marcas convencionales en M. lenticulatus en Nueva Zelanda. En el mismo trabajo incorpora en la discusión resultados en otra especie de Australia, M. antarticus, obtenidos con la misma metodología. Las mayores velocidades de natación estimadas en $M$. lenticulatus fueron similares a las mayores estimadas para M. schmitti. La mayor distancia en $M$. antarticus fue de $2.200 \mathrm{~km}$, escala comparable con los resultados en $M$. lenticulatus. En el gatuzo, la mayor distancia estimada fue menor a 600 $\mathrm{km}$, sin diferencias en las distancias entre los sexos. Tanto en $M$. antarticus como en M. lenticulatus, las hembras fueron recapturadas a mayores distancias que los machos. Las diferencias en la velocidad y en las distancias entre estas especies del mismo género eran esperables ya que $M$. 
lenticulatus presenta mayores tallas en ambos sexos que M. schmitti (Hozbor et al. 2010). Debido al sesgo en la distribución del esfuerzo, no fue posible establecer el hábitat invernal de $M$. lenticulatus, ya que la mayor parte de las recapturas ocurrieron durante el verano (Francis 1988). Sin embargo, el autor señala que pasaría el invierno en aguas del talud o aún a mayores profundidades mientras habitaría la plataforma de Nueva Zelanda en primavera y verano. No descarta la posibilidad de que en invierno permanezca en la plataforma aunque se disperse en un área mayor. Según este autor, los desplazamientos de ambos sexos estarían vinculados al ciclo reproductivo anual, al igual que en otras especies correspondientes al mismo género, pero no descarta que las hembras también podrían agregarse en la costa para obtener mediante el alimento algunos componentes esenciales no disponibles en su hábitat invernal. En el caso del gatuzo, se marcaron ejemplares en otoño (Mayo) y se los recapturó en invierno a los 90 y 100 días, tanto en la Zona Norte como en la Sur, por fuera de la temporada reproductiva de la especie. Esto indica que los desplazamientos no solo estarían vinculados con la reproducción sino también con otros factores por ahora desconocidos.

Los resultados sobre la marcación de gatuzo pusieron en evidencia la utilización de áreas extensas mayoritariamente costeras (principalmente profundidades menores a $50 \mathrm{~m}$ ) con una proporción importante de individuos (juveniles $>47 \mathrm{~cm}$ de talla y adultos) sin alta afinidad con algunas áreas particulares. Este fue el modelo propuesto por Knip et al. (2010) a escala mundial para tiburones pequeños. Sin embargo, como ya había sido discutido por Oddone et al. (2005) y Cortés et al. (2011), la migración y agregación reproductiva en primavera-verano, la utilización de algunas áreas reducidas como áreas de cría (Colautti et al. 2010) con presencia de individuos adultos para parir y luego aparearse y su posterior desaparición de esas áreas en otoño-invierno (Oddone et al. 2007; Colautti et al. 2010; Cortés et al. 2011; Elisio et al. 2015), indicaría un ajuste al modelo propuesto por Springer (1967), habitualmente señalado en el caso de grandes tiburones. Por lo tanto, el caso del gatuzo parece contener algunos aspectos correspondientes a ambos modelos, un modelo mixto entre los ya señalados, con fidelidad por los sitios de reproducción pero sin evidencias de filopatría.

Contrubución INIDEP Nº 2209.

\section{REFERENCIAS}

Acha EM, Mianzan HW, Guerrero RA, FAVERo M, BAVA J. 2004. Marine fronts at the continental shelves of austral South America. Physical and ecological processes. J Marine Syst. 44 (1/2): 83-105.

Acha EM, Piola AR, Iribarne OO, Mianzan HW. 2015. Ecological processes at marine fronts: oases in the ocean. Springer Briefs In Environmental Science. Cham: Springer. 68 p.

AndReoli HG, TRUCCO MI. 2015. Diversidad y estructura genético poblacional de Mustelus schmitti mediante Inter Simple Sequence Repeats (ISSR). Inf Invest INIDEP $\mathrm{N}^{\circ}$ 18/2015. 50 p.

Andreoli HG, Trucco MI. 2018. Diversidad y estructura genético poblacional de Mustelus schmitti mediante Inter simple sequence repeats (ISSR). Frente Marít. 25: 275-291.

Becker JJ, Sandwell DT, Smith WH, Braud J, Binder B, Depner J, FABre D, Factor J, IngALlS S, KIM S-H, et al. 2009. Global Bathymetry and Elevation Data at 30 Arc Seconds Resolution: Srtm30_Plus. Mar Geod. 32 (4): 355-371.

Bellegia M, Figueroa DE, Sanchez F, Bremec C. 2012. The feeding ecology of Mustelus schmitti in the Southwestern Atlantic: geographic variations and dietary shifts. Environ Biol Fish. 95 (1): 99-114.

Brevé NW, Winter HV, VAN OVERZEe HM, FAR- 
RELL ED, WALKer PA. 2016. Seasonal migration of the starry smooth-hound shark Mustelus asterias as revealed from tag-recapture data of an angler-led tagging programme. J Fish Biol. 89: 1158-1177.

Brownie C, ANderson K, Burnham K, Robson D. 1985. Statistical inference fron band recovery data: a handbook. USFWS Resource Publication 156.

Campos BR, Fish MA, Jones G, Riley RW, Allen PJ, Klimley PA, Kelly JT. 2009. Movements of brown smoothhounds, Mustelus henlei, in Tomales Bay, California. Environ Biol Fish. 85 (1): 3-13.

Chapman DD, Feldheim KA, Papastamatiou YP, HUETER RE. 2015. There and back again: A review of residency and return migrations in sharks, with implications for population structure and management. Annu Rev Mar Sci. 7: 547-570.

Colautti D, Baigun C, Cazorla Al, Llompart F, Molina JM, Suquele P, Calvo S. 2010. Population biology and fishery characteristics of the smooth-hound Mustelus schmitti in Anegada Bay, Argentina. Fish Res. 106 (3): 351-357.

Colonello J, Christiansen H, Macchi G. 2007. Escala de madurez sexual para peces cartilaginosos en la Plataforma Continental Argentina. Inf Téc INIDEP No 74/2007. 15 p.

CORTÉs F. 2007. Sustentabilidad de la explotación del gatuzo, Mustelus schmitti en el ecosistema costero bonaerense $\left(34^{\circ}-42^{\circ} \mathrm{S}\right)$ [tesis de licenciatura]. Mar del Plata: Facultad de Ciencias Exactas y Naturales, Universidad Nacional de Mar del Plata. 38 p.

Cortés F, Hozbor N, Pérez M, Massa A. 2016. Aplicación de un Modelo de Dinámica de Biomasas al gatuzo (Mustelus schmitti) en el área del Tratado del Río de la Plata y su Frente Marítimo (1983-2015). Doc. Trab. INIDEP N ${ }^{\circ}$ 17/2016. 14 p.

Cortés F, Jaureguizar AJ, Menni RC, GueRRERO RA. 2011. Ontogenetic habitat prefer- ences of the narrownose smooth-hound shark, Mustelus schmitti, in two southwestern Atlantic coastal areas. Hydrobiologia. 661 (1): 445-456.

Cousseau MB. 1986. Estudios biológicos sobre peces costeros con datos de dos campañas de investigación realizadas en 1981. VI. El gatuzo (Mustelus schmitti). Frente Marít. 1 (1): 60-65.

Cousseau MB, Carozza CR, Macchi GJ. 1998. Abundancia, reproducción y distribución de tallas del gatuzo (Mustelus schmitti). En: LASTA C, editor. Resultado de la campaña EH13/94. INIDEP Inf Téc. 21: 103-115.

Cousseau MB, Perrotta RG. 2013. Peces marinos de Argentina. Biología, distribución, pesca. Mar del Plata: Instituto Nacional de Investigación y Desarrollo Pesquero (INIDEP). $193 \mathrm{p}$.

Cuevas M, Cedrola P, Chiaramonte G. 2013. Manual de marcado de tiburones de Argentina; [consultado 2 octubre 2019]. https://www. reearchgate.net/publication/258565077_Man ual_de_marcado_de_Tiburones_de_Argentina /link/02e7e528a62dd84fd5000000/download.

da Silva C, Kerwath SE, Attwood CG, Thorstad EB, Cowley PD, Økland F, Wilke CG, NÆSJE TF. 2013. Quantifying the degree of protection afforded by a no-take marine reserve on an exploited shark. Afr J Mar Sci. 35 (1): 57-66.

Elisio M, Colonello J, Massa A, Cortés F, Jaureguizar A, Hozbor N, Macchi G. 2015. Eventos reproductivos poblacionales de gatuzo (Mustelus schmitti) y su relación con la temperatura y la profundidad en aguas del sur de la provincia de Buenos Aires. Inf Invest INIDEP N ${ }^{\circ}$ 65/2015. 12 p.

EsPinOZA M, FARrugia TJ, Lowe CG. 2011. Habitat use, movements and site fidelity of the gray smooth-hound shark (Mustelus californicus) in a newly restored Southern California estuary. J Exp Mar Biol Ecol. 401 (1-2): 63-74.

FRANCIS M. 1988. Movement patterns of rig 
(Mustelus lenticulatus) tagged in southern New Zealand. New Zeal J Mar Fresh. 22: 259272.

González Carman V, Mandiola A, Alemany D, Dassis M, Seco Pon JP, Prosdocimi L, Ponce de Leon A, Mianzan H, Acha M, Rodriguez D, et al. 2016. Distribution of megafaunal species in the southwestern atlantic: Key ecological areas and oportunities for marine conservation. ICES J Mar Sci. 73 (6): 1579-1588.

Hilborn R, Walters C. 1992. Quantitative fisheries stock assessment. Choice, dynamics and uncertainty. Nueva York: Chapman \& Hall. $570 \mathrm{p}$.

Hoenig J, Barrowman N, Hearn W, Pollock H. 1998a. Multiyear tagging studies incorporating fishing effort data. Can J Fish Aquat Sci. 55: 1466-1476.

Hoenig J, Barrowman N, Pollock H. 1998 b. Models for tagging data that allow for incomplete mixing of newly tagged animals. Can J Fish Aquat Sci. 55: 1477-1483.

Hozbor N, Massa A. 2015. Análisis de las capturas y de la actividad de la flota con declaraciones de condrictios. Periodo 1992-2014. Inf Invest INIDEP N ${ }^{\circ}$ 64/2015. 13 p.

Hozbor N, SAEz M, Massa A. 2010. Edad y crecimiento de Mustelus schmitti en la región costera bonaerense y uruguaya. Inf Invest INIDEP N ${ }^{\circ}$ 49/2010. 15 p.

[INIDEP] InSTITUTO NACIONAL DE INVESTIGACIÓN y Desarrollo Pesquero. 2015. Gabinete de Oceanografía Física - Base Regional de Datos Oceanográficos (BaRDO). Ministerio de Agricultura Ganadería y Pesca, Subsecretaría de Pesca y Acuicultura de la Nación. Instituto Nacional de Investigación y Desarrollo Pesquero. Paseo Victoria Ocampo $N^{o} 1$, Mar del Plata, Bs. As. Argentina.

Irigoyen A, Cristiani F, Sibbald C, Trobbiani G. 2015. Patrones estacionales de abundancia en el Golfo Nuevo y migración a lo largo de la plataforma Argentina de cazones (Galeorhi- nus galeus) y gatopardos (Notorynchus cepedianus) (Argentina). Ecología Austral. 25: 144-148.

Jaureguizar A, Wiff R, Clara ML. 2016. Role of the preferred habitat availability for small shark (Mustelus schmitti) on the interannual variation of abundance in a large Southwest Atlantic Coastal System (El Rincón, 39$41^{\circ} \mathrm{S}$ ). Aquat Living Resour. 29 (3): 305-310.

KNIP DM, Heupel MR, Simpfendorfer CA. 2010. Sharks in nearshore environments: models, importance, and consequences. Mar Ecol Prog Ser. 402: 1-11.

KoHLER NE, Turner PA. 2001. Shark tagging: a review of conventional methods and studies. Environ Biol Fish. 60: 191-223.

lasta C, Ruarte C, Carozza C. 2001. Flota Costera Argentina: antecedentes y situación actual. En: Bertolotti MI, Verazay GA, Akselman R, editores. El Mar Argentino y sus recursos pesqueros. Tomo 3. Evolución de la flota pesquera argentina, artes de pesca y dispositivos selectivos. Mar del Plata: Instituto Nacional de Investigación y Desarrollo Pesquero (INIDEP). p. 89-119.

Lucas A, Guerrero R, Mianzan H, Acha M, LASTA C. 2005. Coastal oceanographic regimes of the Northern Argentine Continental Shelf $\left(34^{\circ}-43^{\circ} \mathrm{S}\right)$. Est Coast Shelf Scien. 65 (3): 405-420.

Lucífora LO, García V, MENni R, Worm B. 2012. Spatial patterns in the diversity of sharks, rays, and chimaeras (chondrichthyes) in the southwest Atlantic. Biodivers Conserv. 21 (2): 407-419.

Massa A, Hozbor N, Chiaramonte G, BalesTRA A, VoOren C. 2006. Mustelus schmitti. The IUCN Red List of Threatened Species 2006: e.T60203A12318268; [consultado 9 marzo 2016]. http://dx.doi.org/10.2305/IUCN. UK.2006.RLTS.T60203A12318268.en.

Massa A, Lasta C, Carozza C. 2004. Estado actual y explotación del gatuzo (Mustelus schmitti). En: SANCHEZ RP, BEZZI S, editores. 
El Mar Argentino y sus recursos pesqueros. Tomo 4. Los peces marinos de interés pesquero. Caracterización biológica y evaluación del estado de explotación. Mar del Plata: Instituto Nacional de Investigación y Desarrollo Pesquero (INIDEP). p. 67-83.

Matano R, Combes V, Piola A, Guerrero R, Palma E, Ted Strub P, James C, Fenco H, Chao Y, Saraceno M. 2014. The salinity signature of the cross-shelf exchanges in the Southwestern Atlantic Ocean: Numerical simulations, J Geophys Res Oceans. 119: 79497968.

McGarvey R, Feenstra J. 2002. Estimating rates of fish movement from tag recoveries: conditioning by recapture. Can J Fish Aquat Sci. 59: 1054-1064.

Menni R, Cousseau M, Gosztonyi A. 1986. Sobre la biología de los tiburones costeros de la provincia de Buenos Aires. Anales de la Sociedad Científica Argentina. 213: 3-26.

[NOAA] National OCEANIC AND ATMOSPheriC Administration. 2014. Boulder, Colorado, USA; [consultado setiembre 2014]. http:// www.esrl.noaa.gov/psd/data/gridded/data. noaa.oisst.v2.html.

Oddone MC, PAesch L, Norbis W. 2005. Reproductive biology and seasonal distribution of Mustelus schmitti (Elasmobranchii: Triakidae) in the Rio de la Plata oceanic front, south-western Atlantic. J Mar Biol Assoc UK. 85: 1193-1198.

Oddone MC, Paesch L, Norbis W, Velasco G. 2007. Population structure, distribution and abundance patterns of the patagonian smoothhound Mustelus schmitti Springer, 1939 (Chondrichthyes, Elasmobranchii, Triakidae) in the Rio de la Plata and inner continental shelf SW Atlantic Ocean (3430'-39³0'S). Braz J Oceanogr. 55 (3): 167-177.

Pereyra S, Garcia G, Miller P, Oviedo S, Domingo A. 2010. Low genetic diversity and population structure of the narrownose shark (Mustelus schmitti). Fish Res. 106 (3): 468-473.
Pereyra I, Orlando L, Norbis W, Paesch L. 2008. Variación espacial y temporal de la composición por tallas y sexos del gatuzo Mustelus schmitti Springer, 1939 capturado por la pesca de arrastre en la costa oceánica uruguaya durante 2004. Rev Biol Mar Oceanogr. 43 (1): 159-166.

Pérez M, Braccini M, Cousseau B. 2014. Uso de marcas convencionales en un país con escasa experiencia en marcación de peces marinos. Lat Am J Aquat Res. 42 (1): 258-263.

Pérez M, Massa A. 2011. Plan de marcación de gatuzo (Mustelus schmitti) en el ecosistema costero bonaerense. Frente Marít. 22: 207-212.

Pollock K, Hoenig J, Jones C. 1991. Estimation of fishing and natural mortality when a tagging study is combined with creel or port sampling in Creel and angler surveys in fisheries management. American Fisheries Society, Symposium 12, Bethesda, MD. p. 423-434.

R CORE TEAM. 2013. R: A language and environment for statistical computing. R. Foundation for Statistical Computing, Vienna, Austria. URL. https://www.R-project.org/.

RAO J. 1967. Large sample tests for the homogeneity of angular data. Sankhya. 28:172-174.

Schlaff A, Heupel M, Simpfendorfer CA. 2014. Influence of environmental factors on shark and ray movement, behaviour and habitat use: a review. Rev Fish Biol Fish. 24 (4): 1089-1103.

SCRIPPS. 2014. UCLA, San Diego, USA: Global Topography; [consultado octubre 2014]. http://topex.ucsd.edu/cgi-bin/get_srtm30.cgi

Sidders M, TAmini L, Pérez J, Chiaramonte G. 2005. Reproductive biology of Mustelus schmitti Springer, 1939 (Chondrichthyes, triakidae) in Puerto Quequen, Buenos Aires Province. Rev Mus Argentino Cienc Nat ns. 7 (1): 89-101.

Silveira S, Pérez M, Mas F, Forselledo R, Cortés F, LAporta M, Domingo, A. 2018. Anexo I. Programa conjunto de marcadorecaptura de gatuzo (Mustelus schmitti) en el 
área del Tratado del Río de la Plata y su Frente Marítimo. Frente Marít. 25: 393-402.

SPRINGER S. 1967. Social organisation of shark populations. En: GILBERT PW, MATHESON RF, RALL DP, editores. Sharks, skates and rays. Baltimore: John Hopkins Press. p. 149-174.

UICN. 2015. The IUCN Red List of Threatened Species. Version 2015-4; [consultado 19 noviembre 2015]. http://www.iucnredlist.org. VOOREN CM. 1997. Demersal elasmobranchs.
En: Seeliger U, Odebrecht C, Castello JP, editores. Subtropical convergence environments: the coastal and sea in the Southwestern Atlantic. Berlin: Springer. p. 141-146.

ZAR JH. 1999. Biostatistical analysis. 4th ed. Upper Saddle River: Prentice Hall.

Recibido: 3-12-2019

Aceptado: 10-3-2020 